\title{
LA FABRICATION DE LA CAPOEIRA DE LONDRES: MESTRE SYLVIA ET
}

\section{LE LSC}

\author{
Daniel Granada ${ }^{1}$
}

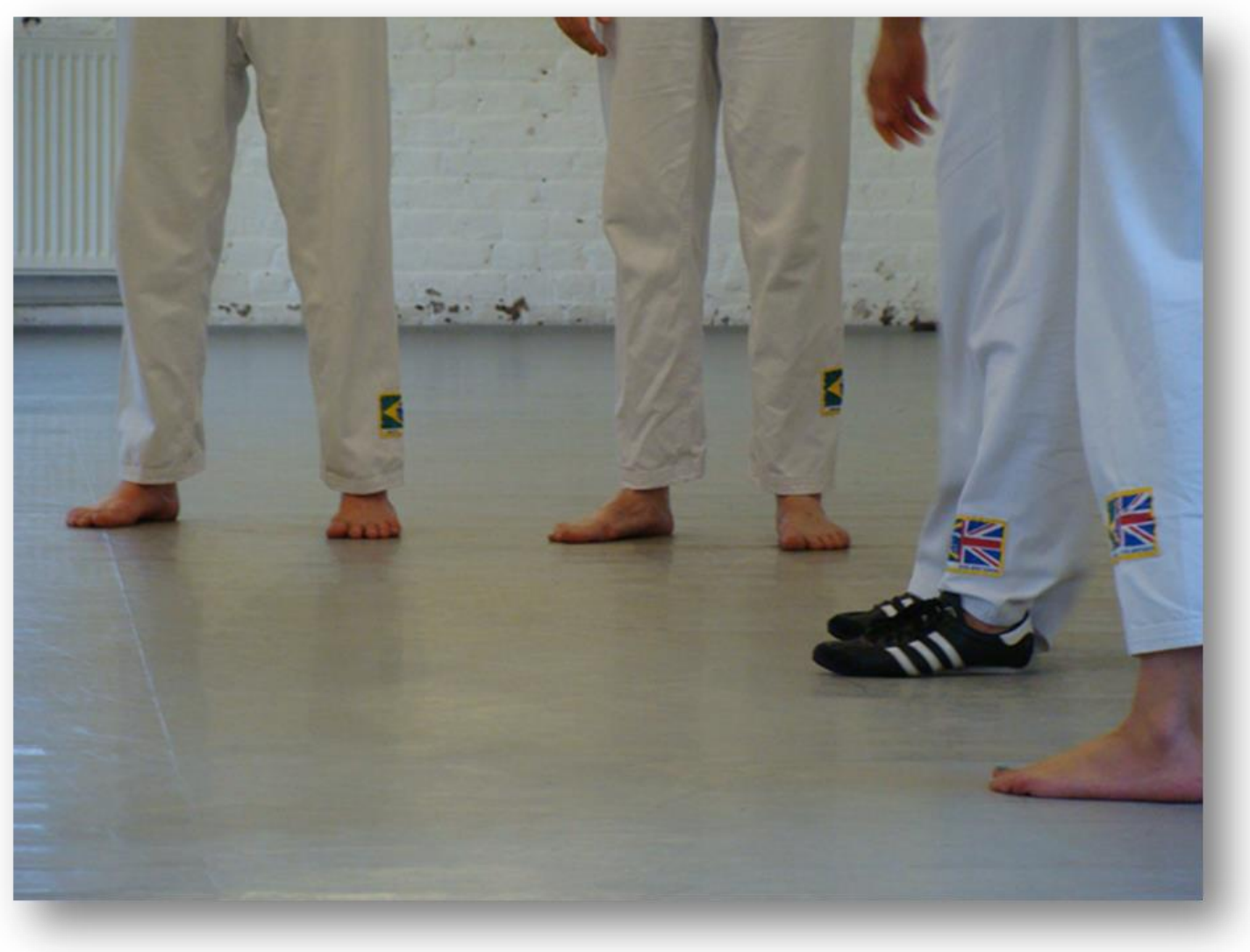

Figure 1: LSC Herança, 2011 (c) Daniel Granada

$\mathrm{La}^{2}$ première vague d'expansion de la capoeira hors du Brésil est redevable en grande mesure aux groupes de spectacles folkloriques qui comprenaient parmi leurs troupes des capoeiristas. Cependant, d'autres capoeiristas, par le biais d'initiatives individuelles et en quête de meilleures conditions de vie et de travail à l'étranger se

\footnotetext{
${ }^{1}$ Université de Paris Ouest Nanterre la Défense, France ; Univates, Brésil.

${ }^{2}$ Cet article a été élaboré à partir d'une thèse de doctorat en Ethnologie et Histoire « Les mestres, les groupes et les"lieux dynamiques" Identité et relocalisation de la pratique de la capoeira à Paris et à Londres » dans le cadre d'une cotutelle entre l'Université de Paris Ouest Nanterre La Défense et l'University of Essex, sous la direction de Stefania Capone et Matthias Röhrig Assunção. Ce travail soutenu en 2013 a été rendu possible grâce à une bourse de cotutelle de thèse accordée par l'Université de Paris Ouest Nanterre La Défense et à une bourse de la Région Ile de France, dispositif SETCI (Soutien à l'encadrement de thèse en cotutelle internationale), la recherche de terrain à Londres à été financée par le Broagan Fund de l'University of Essex et une bourse accordée par la Maison d'Archeologie et Ethnologie, René-Ginouvès.
} 
lancent vers les pays du Nord et découvrent dans la pratique de la capoeira une éventuelle source de revenus. Assunção (2005: 187-8) signale que le premier à enseigner la capoeira sur le vieux continent a sûrement été Mestre Nestor Capoeira ${ }^{3}$. Arrivé à Londres en 1971, il donne des cours de capoeira pendant un an dans une académie de danse puis il sillonne l'Europe durant trois ans au cours desquels il œuvre dans différentes villes, avant de rentrer au Brésil. L'article analysera l'installation d'un des premiers groupes de capoeira à Londres, c'est-à-dire celui que l'on peut considérer comme l'un des pionniers dans le processus de consolidation de la capoeira au Royaume Uni. Les femmes jouent un rôle primordial dans la naissance des premières écoles de capoeira officielles dans la ville, la LSC de Mestre Sylvia est un example concret que le femmes ont joué un role central dans le processus de transnationalisation de la pratique de la capoeira. Assunção (Idem: 180) souligne le que fait que dans les années 1990, très peu de femmes participent aux groupes de capoeira de Bahia, alors dans les états du Sud du Brésil et aux États-Unis la participation des femmes était plus fréquente. Barbosa (2005: 14) signale au moins 14 facteurs qui expliquent la plus grande participation des femmes dans la capoeira à partir des années 1980, parmi eux: l'augmentation de la classe moyenne au Brésil, la plus grande émancipation des femmes grâce aux mouvements féministes, la place prise par la négritude dans les médias, la pénétration de la capoeira dans les écoles, l'impact positif des capoeiristas auprès des milieux universitaires, le numéro croissant des publications sur la capoeira.

Nonobstant l'environnement majoritairement masculin de la capoeira au Brésil dans les années 1980 et 1990, le développement de la capoeira à Londres s'est déroulé d'une forme inaccoutumée. Ce sont en effet, les femmes qui sont en grande partie les véritables responsables de l'installation des premiers groupes de capoeira dans la capitale anglaise ${ }^{4}$. Je considère qu'il est possible de parler de consolidation de l'expansion de la capoeira au Royaume-Uni seulement à partir de la création des premières écoles de capoeira. Dans ce sens, les cours réguliers de capoeira à Londres s'établissent avec la création de la première école officielle en 1988 sous la direction de Sylvia Bazzarelli, originaire du groupe Senzala de Mestre Sombra de la ville de Santos

\footnotetext{
${ }^{3} \mathrm{Au}$ long de cet article j'emploie la forme brésilienne «mestre » au lieu de master qui serait la traduction littérale en anglais, une fois que les pratiquants de capoeira en Angleterre emploient le mot en portugais, parce que, selon eux le mot master est chargé d'une signification associée à l'esclavage, avec le maître d'esclaves.

${ }^{4}$ D'autres mestres de capoeira comme Mestre Ousado et Mestre Gato ont certainement été importants dans le processus d'implantation des premiers groupes de capoeira au Royaume-Uni (cf. Assunção, 2005: 188), mais l'objectif ici c'est de mettre en évidence l'importance des femmes dans ce processus.
} 
dans l'état de São Paulo 5 .

Cet article à été élaboré à partir d'une thèse soutenue en 2013. La recherche de terrain a été réalisée en France et au Royaume-Uni entre 2007 et 2011, par le biais de l'observation participante, avec l'utilisation de l'ethnographie multi-site (Marcus, 1995; 2002) ou translocal (Hannerz, 2003), etnographie virtuelle (Hine, 2000), des interviews informelles et plus de soixante-dix interviews formelles enregistrées avec les capoeiristas de différents groupes de Paris et sa banlieue et Londres. La recherche visait à appréhender les transformations opérées par les capoeiristas sur la pratique de la capoeira dans ce processus de délocalisation de la pratique brésilienne et sa conséquente réappropriation par des pratiquants en Europe. L'article analyse les rapports de pouvoir entre les pratiquants de la capoeira hors du Brésil à partir de l'analyse du récit de vie d'une femme mestre de capoeira à Londres. L'analyse détaillée de la trajectoire de Mestre Sylvia permettra de mieux appréhender la façon dont les rapports de pouvoir affectent l'organisation de la capoeira hors du Brésil et mettra en lumière les mécanismes utilisés dans la construction de l'authenticité et de la légitimité au sein des groupes, ainsi que les rapports établis envers les autres groupes dans le «marché de la capoeira $\gg 6$.

Reprenant les critiques de Bourdieu (1986) sur « l'illusion biographique » qui remarque que tout le récit de vie est constitué à partir du contexte présent où l'interviewé, avec la complicité de son interlocuteur, cherche à donner du sens de manière cohérente et totalisant à un récit de vie (Bourdieu, 1986: 71). La méthode employée ici cherche, par le biais de l'analyse du récit de vie à expliciter les enjeux liés à leur légitimité en tant que pratiquants de capoeira, met en évidence la capacité des acteurs prendre des décisions et d'agir (agency) dans un contexte transnational.

\footnotetext{
${ }^{5}$ Une autre femme comptant dans l'installation de la capoeira à Londres est Rilene du groupe Amazonas. Professora Rilene comme elle tient à se faire appeler, a un parcours assez différent de celui de Mestre Sylvia, puisqu'elle est issue d'un milieu modeste de la région du Nordeste du Brésil et est arrivée à Londres deux ans après Mestre Sylvia et son mari, un mestre de capoeira (cf. Ferreira, 2015: 165 - 176).

${ }^{6}$ Le « marché de la capoeira », fait référence à un espace structuré de positions, qui sont déterminées par la distribution de différentes espèces de ressources ou de «capital » (Bourdieu, 2002: 113-20). Bourdieu signale que « pour qu'un champ marche, il faut qu'il y ait des enjeux et des gens prêts à jouer le jeu, dotés de l'habitus impliquant la connaissance et la reconnaissance des lois immanentes du jeu, des enjeux, etc » (Idem: 114). Levitt et Glick-Schiller utilisent le terme « champ social » comme « un ensemble de réseaux interconnectés de relations sociales à travers lesquels des idées, des pratiques et des ressources sont échangées, organisées et transformées de façon inégale » (2004: 1009). Cependant Glick-Schiller précise que la notion de « champ social transnational » qu'elle utilise n'est pas inspirée de la notion de Bourdieu (2002), mais du concept de « champ social », développé par l'anthropologie sociale et par les géographes, qui met en avant les rapports sociaux qui relient et transforment les espaces sociaux historiquement basés sur la territorialité, comme la communauté locale, la ville, ou l'État (Glick-Schiller, 2010: 112).
} 
Les photos ici utilisées ont été prises avec l'accord des mestres et des élèves, elles sont disposés au long du texte. L'usage des photos dans cet article n'a pas pour objectif de « dire la vérité » sur le terrain (Becker, 2007), mais plutôt d'élargir le champ de vision et la perception de l'anthropologue (Conord, 2007: 21) et par conséquent du lecteur sur les relations sociales observées, avec la présentation d'images du terrain qui dialoguent avec l'analyse développée ${ }^{7}$.

\section{La capoeira hors du Brésil}

Les premiers à avoir signalé la croissance de la capoeira hors du Brésil sont ceux ayant étudié l'émigration des Brésiliens vers les États-Unis ( $c f$. Margolis 1994, Ribeiro 1999, Penha 2001, Martes 2003). Un grand nombre de Brésiliens qui ont émigré en quête de meilleures conditions de vie et de travail entre les années 1980 et les années 2000 ont emporté avec eux la pratique de la capoeira. Ils ont vu en cette pratique un moyen de survivre tout en restant en contact avec le Brésil.

L'émigration des Brésiliens aux États-Unis est un mouvement migratoire caractérisé, entre autres, par l'importance accordée aux réseaux sociaux tissés par les Brésiliens dans l'explication de ce phénomène (Martes 1999, Margolis 2003, Patarra 2005). Une recherche réalisée à Boston en 1995 démontre que les Brésiliens qui optent pour émigrer vers les États-Unis partent en quête d'opportunités de travail et principalement de l'ascension sociale qui leur a été refusée au Brésil (Sales 1999: 33). L'émigration des Brésiliens vers les États-Unis serait une immigration de «classe moyenne », car elle rend nécessaire certaines ressources qui garantissent l'achat du billet d'avion (Sales, 2005).

La crise économique des années 1980 au Brésil, appelée la « décennie perdue », est vue comme le jalon décisif qui a déclenché l'émigration des Brésiliens. Sales (1999: 28) ajoute le facteur politique associé principalement aux espoirs et désenchantements de la période de redémocratisation du pays traversé par l'échec des divers plans économiques, l'augmentation du chômage et de l'inflation. Patarra (2005) explore

\footnotetext{
${ }^{7}$ Becker explique qu'il faut considérer le rôle d'interaction du lecteur avec la photo: « Il s'ensuit que pour savoir si les images disent la vérité, la première étape consiste à déterminer quelle vérité elles affirment en y repérant les réponses aux questions que nous avons posées ou qu'elles ont elles-mêmes suggérées. (Voir les choses de cette manière met l'accent sur le fait que les images ne se contentent pas d'émettre des affirmations, mais que nous interagissons avec elles pour en tirer des conclusions - en résumé, nous jouons un rôle actif dans ce processus [...]) »(Becker, 2007: 36)
} 
l'hétérogénéité des mouvements migratoires à partir du Brésil et vers le Brésil, et signale l'existence de divers phénomènes et de divers groupes sociaux qui immigrent. L'auteur remarque la nécessité de prendre en compte dans l'explication des phénomènes migratoires du Brésil contemporain l'importance de la circularité de ces mouvements ainsi que les réseaux sociaux qui se constituent et se renforcent (Patarra, 2005: 25-26).

Dans les études sur les Brésiliens aux USA, la capoeira, la samba et la batucada sont souvent présentées comme des manifestations de «brésilité » ou «afro-brésilité » ${ }^{8}$. Cependant, quelques études ont conclu que la participation des Brésiliens au sein de ces groupes n'était pas très représentative en nombre d'adhérents (Margolis, 1994: 307). Les groupes de capoeira et de batucada sont, hors du Brésil, des groupes d'expression de la «brésilité » qui pour la plupart ne sont pas intégrés par des Brésiliens mais par la population locale. En général, seuls les enseignants et un petit nombre restreint de Brésiliens font partie de ces groupes, malgré la grande présence d'immigrés provenant du Brésil aux États-Unis9.

Dans une étude de référence sur les immigrants brésiliens à New York, Margolis (1994) note l'absence de «liens communautaires » au sein de la «communauté des Brésiliens » de New York $^{10}$. Cette constatation a été faite lors de l'observation des activités d'un centre de promotion de la « culture brésilienne » à New York:

Um outro exemplo da ausência de entrosamento comunitário é a falta de apoio para as aulas de especialidades tradicionalmente brasileiras tais como a "capoeira" e a "batucada", oferecidas por aquele centro. Estas aulas, e outras similares oferecidas de forma particular, atraíram apenas alguns entre os milhares de brasileiros que residem na cidade de Nova York. Por exemplo, entre as dezenas de alunos das aulas de

\footnotetext{
${ }^{8}$ Les batucadas sont des orchestres musicaux composés par des instruments de percussion, des tambours de taille variable qui jouent des rythmes « afro-Brésiliens ». La samba est un type de musique aux origines « afro-brésiliennes » très apprécié au Brésil. La «brésilité » ou l'« afro-brésilité » sont des manifestations issues des pratiques populaires brésiliennes qui sont devenues des symboles du pays et qui sont identifiées comme des pratiques du Brésil à l'étranger. Ces pratiques se sont diffusées principalement à travers les spectacles folkloriques, et par l'initiative des Brésiliens qui décident de rester à l'étranger et d'offrir des cours de ces modalités. Le cas de la capoeira est analysé par Assunção (2005: 185-189) qui remarque l'importance des spectacles folkloriques dans la diffusion de la capoeira aux États-Unis et en Europe.

${ }^{9}$ Un article de Sarah Barrell publié par The Independent le 01/07/2001 intitulé « Capoeira The mysterious South American martial art now has devotees all over the UK » souligne la présence de deux capoeiristas brésiliens parmi tous les élèves d'origines diverses du cours auquel elle avait assisté au London School of Capoeira.

${ }^{10}$ L'absence d'un sentiment d'appartenance communautaire entre les migrants brésiliens aux États-Unis est expliquée par Margolis (2003: 59) par le fait que les Brésiliens vont aux États-Unis pour travailler et gagner un maximum d'argent sur une courte période. Comme ils cumulent deux ou trois emplois, ils n'ont pas le temps de rejoindre des organisations communautaires. Les Brésiliens, d'après l'auteur, renient leur condition d'immigré et affirment qu'ils sont aux États-Unis ponctuellement, dans l'intention de rentrer dès que possible dans leur pays.
} 
« capoeira » oferecidas em Nova York, desde meados dos anos 1970, apenas cinco dos matriculados são atualmente brasileiros. Entre os frequentadores das escolas de samba locais, 90 por cento não eram brasileiros; (...). Aparentemente, estes grupos são integrados por americanos aficionados da cultura e da música brasileiras, mais do que brasileiros envolvidos com sua herança cultural (Margolis, 1994: 307).

Ce qui est interprété par Margolis comme un exemple d'absence de liens communautaires, ou un manque d'intérêt envers les spécialités brésiliennes, constitue en effet l'une des caractéristiques principales du processus d'implantation de la capoeira à l'étranger. Lors de ma recherche de terrain, la majeure partie des pratiquants de capoeira, dans les groupes que j'ai étudiés en France et au Royaume-Uni, ne sont pas brésiliens $^{11}$. De façon générale, le nombre de Brésiliens n'est pas très important dans les groupes de capoeira hors du Brésil, même dans des villes où l'immigration brésilienne est considérable comme à Paris et à Londres. Si la présence des femmes en tant qu'élèves est notable dans les deux pays, les femmes comme leaders de groupes ont été trouvés seulement au Royaume Uni.

Après cette première vague d'études sur l'immigration des Brésiliens aux ÉtatsUnis, deux études ont été consacrées à l'analyse de la pratique de la capoeira et de son appropriation hors du Brésil: les études pionnières de Travassos (2000) sur la capoeira aux États-Unis et celles de Vassallo (2001) sur la capoeira en France.

Les théories sur les migrations internationales occupent une place centrale dans l' explication de l'expansion de la pratique de la capoeira hors du Brésil. Des travaux récents, comme celui d'Aceti (2011) sur l'expansion de la capoeira en Europe et la thèse de Guizardi (2011) qui traite de la capoeira en Espagne et à Madrid, montrent l'importance accordée aux théories sur l'immigration pour expliquer l'expansion de la capoeira en Europe. Certaines de ces études, comme celle de Delamont et Stephens (2008: 61), reconnaissent l'importance de la migration des Brésiliens pour comprendre la capoeira hors du Brésil, sans toutefois analyser la bibliographie sur les Brésiliens migrants ni les spécificités de cette migration. D’autres comme celle de Joseph (2008: 197-199) sur la capoeira au Canada, et de Guizardi (2011: 365-414) à propos de la capoeira à Madrid, remarquent l'importance de l'immigration des Brésiliens pour l'explication de la croissance de la capoeira dans leur pays respectif. La thèse d'Aceti

\footnotetext{
${ }^{11}$ La recherche effectuée dans le cadre de ma thèse s'est concentrée sur deux groupes en France: l'association Kolors de Paris et le groupe Ypiranga de Pastinha d'Ivry-sur-Seine. Au Royaume-Uni, la recherche a été ciblée sur les activités de cinq groupes londoniens: London School of Capoeira, Amazonas, East London Capoeira, Cordão de Ouro Londres et Kabula.
} 
reconnait le rôle de l'immigration des pratiquants brésiliens mais met en évidence la participation et l'importance de pratiquants non-brésiliens en Europe aussi bien que le role des femmes dans les groupes de capoeira et leur importance pour le processus de transnationalisation de la pratique (Aceti 2011: 234-236; 441) ${ }^{12}$.

Ces études mettent en évidence les questions des rapports entre le «culturel » et le « politique » au sein des divers contextes nationaux, aussi bien que les implications identitaires et les rapports de pouvoir issus de la rencontre entre les Brésiliens et les pratiquants locaux au Canada (Joseph, 2008, 2008a), en France (Ferreira, 2005, 2007, 2008, 2010; 2015; 2015a; Gravina, 2010; Brito, 2011) et en Espagne (Guizardi, 2011, 2013) . Récemment des études ont mis en lumière l'impact de la transnationalisation de cette pratique au Brésil, avec les exigences croissantes sur la légitimité et la professionnalisation des enseignants et mestres de capoeira (Wesolowski, 2012).

Dans cet article je présente le parcours exemplaire de Mestre Sylvia, ensuite j'analyserai la creation de son groupe de capoeira à Londres et les conditions dans lesquelles il s'est développé. En me basant sur ma recherche de terrain, j'analyserai les points importants qui la permis de s'imposer, sur le «marché de la capoeira » de Londres. Il s'agit de montrer de quelle façon elle a construit sa légitimité ainsi que le rapport avec les autres groupes afin de s'imposer dans ce marché. Ainsi, cette démarche permettra de comprendre les logiques de resémantisation de la capoeira pour l'adapter au public local, aussi bien que les réinterprétations individuelles et collectives, fruits des besoins de ce marché et de la circulation des capoeiristas en Europe et au Brésil. Le besoin de légitimation de ces groupes dans le contexte local entraîne des alliances et des ruptures avec les groupes sur place et au Brésil. L'analyse détaillée de la trajectoire de Sylvia permettra de mieux appréhender la façon dont les rapports de pouvoir affectent l'organisation de la capoeira hors du Brésil et mettra en lumière les mécanismes utilisés dans la construction de l'authenticité et de la légitimité à l'intérieur de ce groupe.

\footnotetext{
${ }^{12}$ L'alliance avec les locaux est notable car quand les groupes sont dirigés par des Brésiliens, il est nécessaire de compter sur les élèves qui jouent le rôle de véritables traducteurs et interprètes pour le contexte local. Il n'est pas rare que les Brésiliens capoeiristas se trouvent, à l'intérieur des groupes, des compagnons qui vont avoir une place importante dans l'organisation du groupe, en y exerçant un rôle de secrétariat et fréquemment en hébergeant le capoeirista et en s'acquittant de ses factures, comme l'a expliqué Aceti (2011: 441).
} 


\section{Mestre Sylvia et la London School of Capoeira Herança (LSC)}

Lors de la recherche de terrain plusieurs capoeiristas ont tenté de me décourager d'aller à la rencontre de Mestre Sylvia et de la LSC. Les commentaires instillaient que la LSC était très renfermée et épiloguaient sur le fait que les élèves ne se mêlaient pas aux autres groupes et que les capoeiristas extérieurs n'étaient pas les bienvenus dans leur espace d'entraînement. Néanmoins, leurs réticences n'ont fait que stimuler ma curiosité. Ce n'est que bien plus tard que j'ai compris que les autres groupes avaient cherché intentionnellement à me décourager parce que la LSC est un des groupes organisés depuis longtemps dans la capoeira à Londres. Ses membres sont, en effet, les premiers à s'être organisés en tant qu'école. Déjà, ils louent un espace exclusivement pour leur activité à Londres, fait assez rare hors du Brésil ${ }^{13}$. De plus, leurs noms ont été cités dans plusieurs reportages sur la capoeira dans les journaux nationaux ${ }^{14}$. Ils ont aussi participé à des publicités pour la marque de téléphone mobile Nokia en 2000 et 2011 et ont organisé des spectacles mélangeant la capoeira et le théâtre dans de prestigieuses salles de spectacle de la capitale londonienne.

Dans un premier temps, je me suis rendu sur leur site internet. C'est bien sur ces pages que les groupes de capoeira organisent leurs discours au sein de la « communauté de la capoeira ». Leur site affiche des informations quant à l'organisation pratique des cours (horaires, locaux, tarifs) mais indique également les lignages d'appartenance des capoeiristas et de leur groupe, les photos des mestres, leur appropriation de l'histoire de la capoeira. Il offre aussi de nombreux articles à la vente destinés aux pratiquants de capoeira. Parmi ces articles sont en vente quatre DVDs et deux CDs du groupe, des tshirts, pantalons et sac-à-dos payables en ligne par carte de crédit. Le lecteur peu habitué aux groupes de capoeira et à leur forme d'organisation peut ne pas comprendre

\footnotetext{
${ }^{13}$ Lors de l'entretien, Mestre Sylvia m'a précisé qu'ils n'ont pas acheté leurs locaux d'entraînement, mais qu'ils ont souscrit à une forme de contrat de location de longue durée qui permet au groupe une plus grande stabilité.

${ }^{14}$ Parmi les articles rencontrés dans la presse anglaise la LSC figure sur les sites internet suivants: Cook, Emma. Adrenalin / Liberty cap: Capoeira's blend of African rhythms and teasing maneuvers is rooted in 15th-century slavery. The Independent [en ligne]. Janvier 1994, [ref. du 17 janvier 1994]. Disponible sur www.independent.co.uk ; Gilbert, Matt. Getting fit for capoeira. The Sunday Times [en ligne]. Février 2007, [ref. du 18/02/2007]. Disponible sur www.thesundaytimes.co.uk/; Murphy, Sam. All you need to know about capoeira. The Guardian [en ligne]. Mars 2007, [ref. du 27 mars 2007]. Disponible sur www.theguardian.co.uk; Barrell, Sarah. Capoeira The mysterious South American martial art now has devotees all over the UK. The Independent [en ligne]. Juillet 2001 [ref. du 1 juillet 2001]. Disponible sur www.independent.co.uk; Allott, Serena. Fighting fit. The Telegraph [en ligne]. Février 2009 [ref. du 20 février 2009]. Disponible sur www.telegraph.co.uk Les vidéos Nokia sont disponibles sur: http://www.youtube.com/watch?v=emtT8Q0T8hw et: http://www.youtube.com/watch?v=q_XjgSC6ST4.
} 
ce qui est exceptionnel dans cette organisation et au sujet de la vente d'objets sur internet, néanmoins, pour les groupes de capoeira dont une grande partie fonctionne d'une manière très informelle et principalement les groupes au Brésil, ces adaptations au marché local sont remarquables et représentent des modes d'organisation novateurs, qui diffèrent de la pratique habituelle.

La LSC se situe dans un ensemble d'anciennes usines. Lorsque j'arrive, la porte est ouverte, mais je frappe tout de même, j'aperçois ensuite Mestre Sylvia derrière une vitre dans un bureau, elle est au téléphone et me fait signe d'attendre. Je profite de ce temps d'attente pour examiner attentivement l'espace de l'école. Dans l'entrée se trouve une armoire comportant des casiers destinés à ranger les vêtements des élèves. Tout est propre et bien entretenu. Un tableau d'affichage met en évidence les règles de l'école, les prix des cours et les autres matériels disponibles à la vente. Du DVD à la corde pour les berimbaus, tout est tarifé de forme claire et précise. À gauche de l'entrée, se trouve une salle secondaire utilisée lorsque les pratiquants sont moins nombreux. À droite, s'ouvre la salle principale plus longue où sont exposées des photos des mestres de capoeira, parmi eux Bimba, Pastinha et bien sûr Mestre Sombra, le fondateur du groupe Senzala de Santos. Sur un tableau, sont épinglés les articles et reportages consacrés à l'école et tirés dans la presse anglaise.

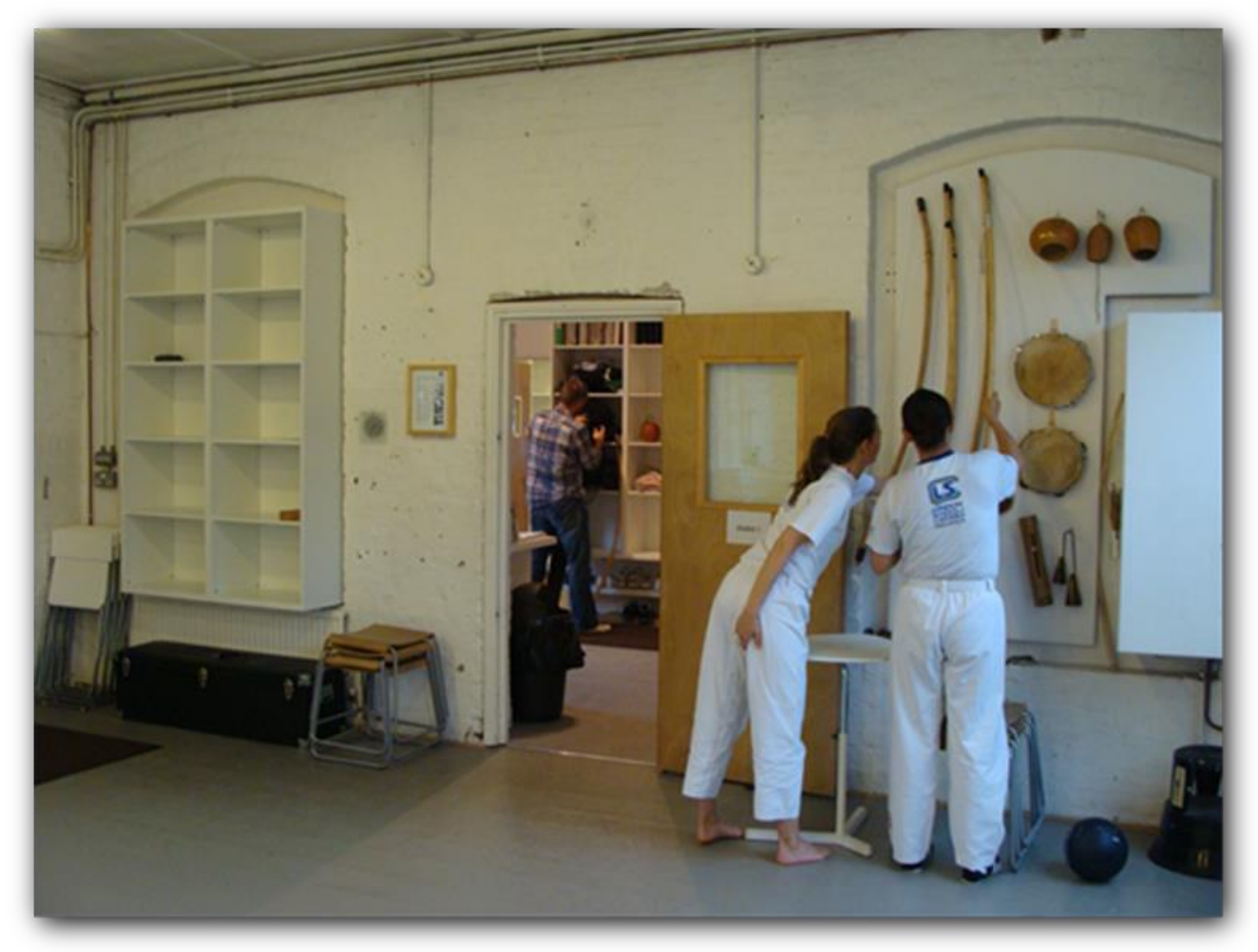

Figure 2: Organisation de l'espace LSC (C) Daniel Granada 


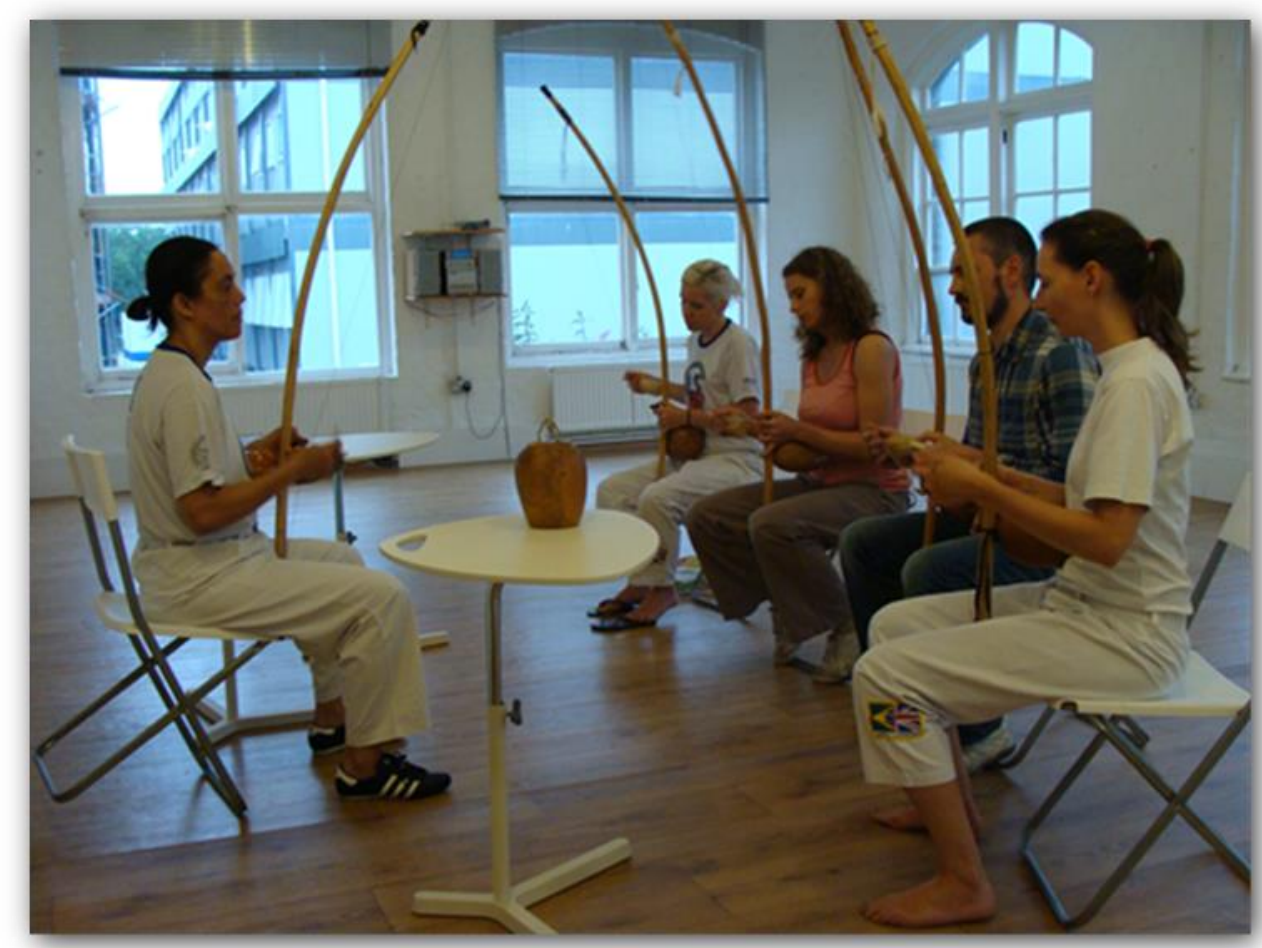

Figure 3: Cours de musique LSC @ Daniel Granada

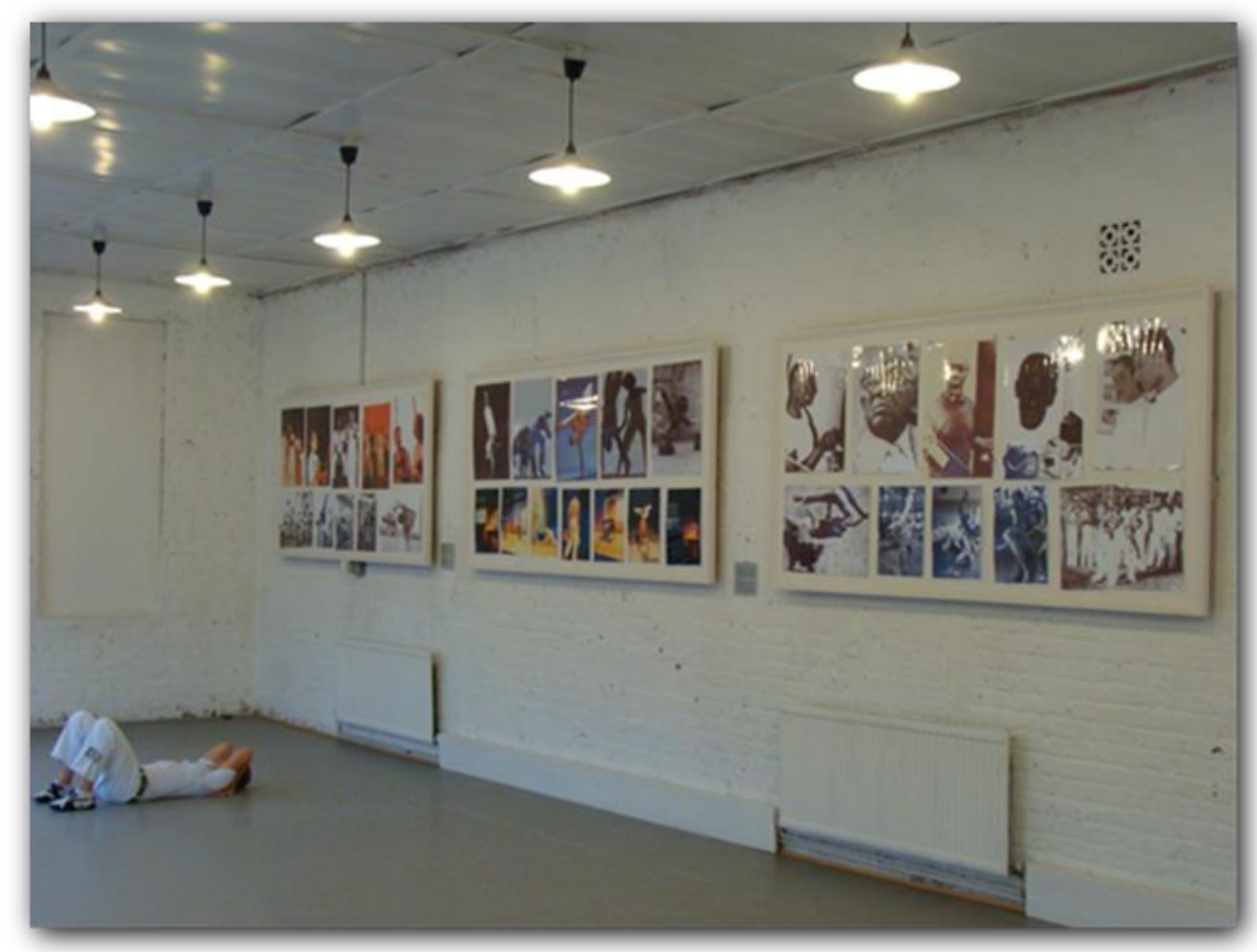

Figure 4: Galerie d'images du groupe LSC @ Daniel Granada 
Face aux portraits des mestres, des berimbaus et pandeiros sont accrochés au mur et une armoire sert à ranger les caxixis, baquetas, pedras et $\mathrm{CDs}^{15}$. Trois atabaques sont rangés au fond de la salle ${ }^{16}$. L'occupation de l'espace par des instruments et des photos de capoeira est importante dans la construction de l'identité collective du groupe $^{17}$. C'est en apprenant à reconnaître les mestres sur les photos et en exhibant les instruments à disposition sur place que l'apprenti commence à se familiariser avec l'espace et les personnages de l'univers de la capoeira. Pouvoir décorer un espace dédié à la pratique de la capoeira avec les photos et les instruments narrant l'histoire du groupe est un véritable défi pour les groupes de capoeira hors du Brésil ${ }^{18}$. Les espaces qu'ils utilisent sont majoritairement occupés de forme transitoire. Les salles sont louées à l'heure et habituellement partagées avec d'autres groupes. L'utilisation partagée des espaces d'entraînements et l'impossibilité de décorer l'espace avec les photos et objets qui constituent la mémoire du groupe confèrent aux sites internet une véritable importance dans la construction de l'identité collective du groupe de capoeira. L'internet et les sites des groupes de capoeira constituent des « lieux » de contact accessibles et permanents avec le groupe de capoeira, puisque la plupart, n'occupe des endroits physiques que de forme éphémère. En ce sens, et dans ce cas précis, l'occupation d'un lieu physique et son appropriation témoignent du pouvoir et de la stabilité du groupe. La LSC est au Royaume-Uni l'un des rares groupes en Europe disposant d'un espace permanent et spécifique pour la pratique de la capoeira. En comparaison avec d'autres groupes rencontrés à Londres, la LSC est le seul à exposer en permanence dans son espace des tableaux et des photos de capoeira. D'autres groupes décorent leurs locaux à l'occasion d'activités extraordinaires lors des stages et des rodas. L'appropriation de l'espace physique et la possibilité au groupe de disposer de l'espace selon les besoins est dans ce sens exceptionnelle et démontre l'importance accordée à un lieu physique dans les groupes de capoeira ${ }^{19}$.

\footnotetext{
${ }^{15}$ Les caxixis sont de petits paniers en paille utilisés pour jouer du berimbau. Les baquetas sont des baguettes en bois servant à frapper la corde du berimbau. Les pedras ou dobrões sont actionnés avec les baguettes pour produire les sons du berimbau.

${ }^{16}$ Les atabaques sont des tambours utilisés lors des rodas de capoeira.

${ }^{17}$ L'usage du terme identité dans ce travail sert à signaler des processus de construction et d'identification fluides et en changement constant au lieu de l'existance des « identités fixes ». Pour un débat sur l'emploi de cette notion par rapport aux groupes de capoeira $c f$. Ferreira, 2015: 49-51.

${ }^{18} \mathrm{Au}$ Brésil aussi, le nombre de groupes disposant d'un espace exclusif pour la pratique de la capoeira est réduit.

${ }^{19}$ Pour la majeure partie des groupes de capoeira avoir un espace d'entraînement fixe qui appartient au groupe est si difficile que certains groupes n'envisage même pas cette hypothèse. Pour d'autres, cela peut devenir un objectif qui lui permettra de se distinguer des autres groupes.
} 


\section{Mestre Sylvia}

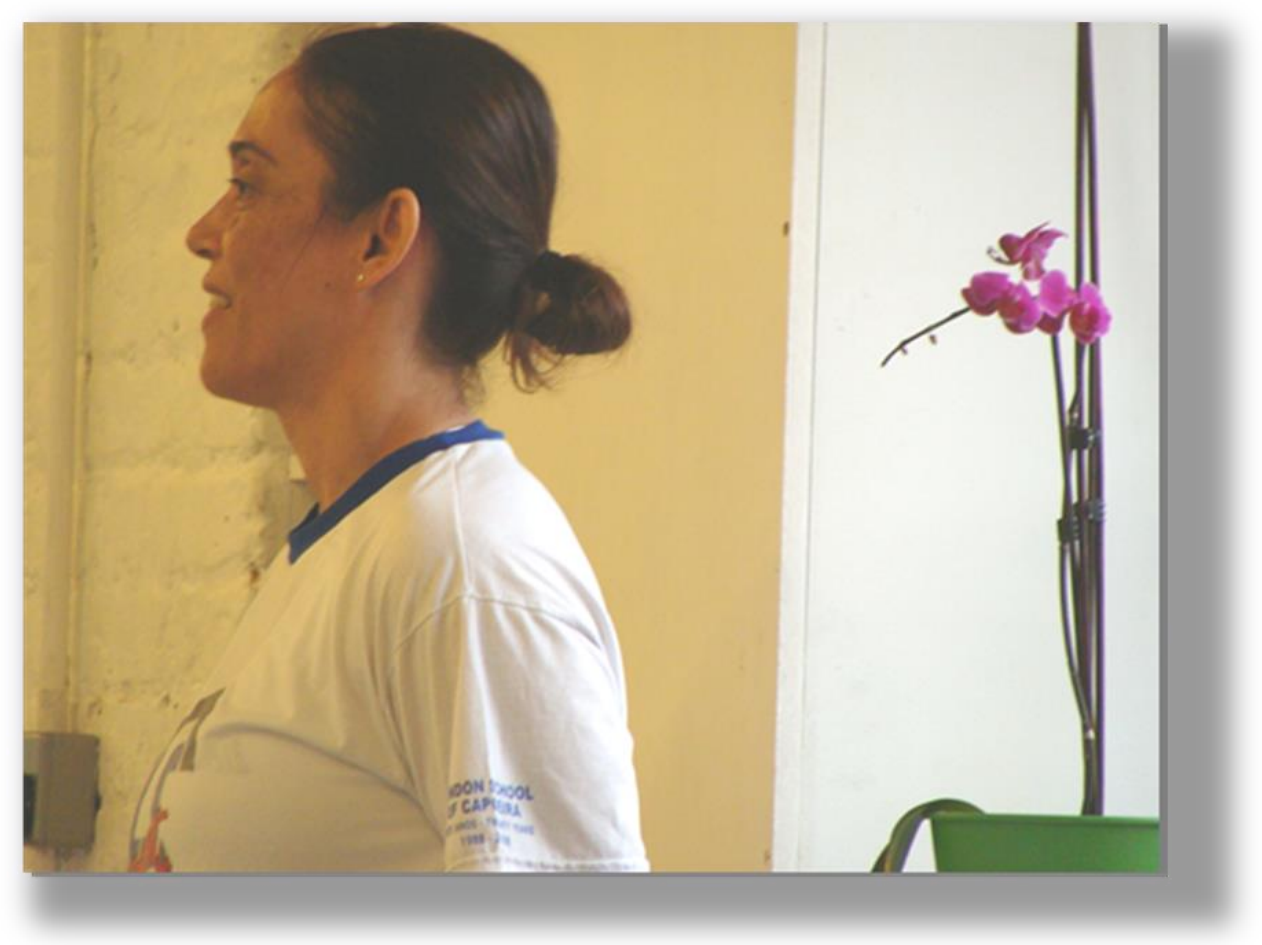

Figure 5: Mestre Sylvia LSC, 2011 C Daniel Granada

Née à Guaratingueta dans l'état de São Paulo, Sylvia est arrivée à Santos à l'âge de cinq ans ${ }^{20}$. Issue d'une famille de classe moyenne, la fondatrice de la LSC rapporte que son père travaillait à Cubatão avant de déménager à Santos avec sa famille en quête d'une meilleure qualité de vie. Mestre Sylvia affirme avoir commencé à pratiquer la danse dès l'âge de huit ans et c'est au lycée qu'une collègue lui a parlé pour la première fois de la capoeira. Elle ne connaissait la capoeira qu'à travers les images véhiculées par la télévision et ajoute qu'à l'époque, il y avait beaucoup de préjugés envers les femmes capoeiristas.

Quelques années plus tard, elle a assisté à un cours d'une association liée au groupe de capoeira Senzala de Santos, dirigé par Mestre Beija-Flor et le professeur Tatu (aujourd'hui Mestre Tatu) puis elle a suivi son premier cours avec ce dernier ${ }^{21}$. Elle est par la suite devenue élève de Beija-Flor. Sylvia explique que c'est parce que Beija-Flor

\footnotetext{
${ }^{20}$ Santos est une ville portuaire de l'État de São Paulo de 419.400 habitants selon les données de l'IBGE (Institut Brésilien de Géographie et Statistique) 2007. C'est le plus grand port d'Amérique Latine et la $18^{\mathrm{e}}$ ville la plus riche du Brésil. Cubatão est une ville industrielle de 118.720 habitants (IBGE, 2007) de l'état de São Paulo connue au Brésil pour des problèmes liés à la pollution.

${ }^{21}$ Mestre Beija-Flor a été l'un des pionniers de la capoeira en France. Selon les dernières informations il est rentré au Brésil récemment.
} 
était élève de Mestre Sombra qu'elle est devenue elle aussi son élève. C'est Sombra qu'elle reconnaît comme son mestre, en dépit du fait qu'elle ait commencé la capoeira avec Beija-Flor. Elle a continué à suivre des cours de danse parallèlement à la capoeira et affirme qu'à cette époque c'était une chose difficile à accepter pour une famille de « classe moyenne »:

«Ils [sa famille] supposaient que j'allais abandonner la danse à la fin du collège. Mais je ne l'ai pas fait et en plus j'ai commencé la capoeira, une pratique inconnue à leur yeux car ils n'avaient pas ce côté artistique dans la famille. Les choses ont commencé à se compliquer pour moi, car j'étais censée passer le Bac et pour cela il fallait suivre le cours de préparation pré-vestibular, mais à cette époque-là j'ai commencé à aider Mestre Sombra lors des cours du matin dans des écoles» ${ }^{22}$.

Sylvia raconte qu'à 19 ans elle était déjà engagée dans diverses activités liées à la capoeira, donnait des cours et avait des responsabilités. Si elle regrette néanmoins de ne pas avoir eu le soutien de sa famille, elle comprend qu'à l'époque il était difficile d'accepter qu'une fille pratique la capoeira. Elle perçoit la difficulté d'acceptation de la part de sa famille comme l'un des motifs de son départ du Brésil ${ }^{23}$. Ayant pratiqué la capoeira dès l'âge de 16 ans, elle ressentait à 22 ans le besoin d'approfondir ses connaissances ; partir à Londres représentait alors la possibilité de concilier la capoeira, le théâtre et la danse loin de sa famille.

\section{L’arrivée à Londres}

Une fois arrivée à Londres, elle s'est sentie désorientée et elle n'a pas tout de suite pensé à enseigner la capoeira. C'est l'envie de continuer à pratiquer et maintenir son art qui l'ont guidée et, progressivement, Sylvia s'est rendu compte qu'il était possible de gagner de l'argent avec la capoeira au lieu de se tourner vers des tâches généralement réservées aux immigrés. Ainsi, elle a débuté à The Place où elle a connu une compatriote qui travaillait au Brazilian Contemporary Art; c'est là qu'elle a obtenu son premier emploi dans la capoeira ${ }^{24}$. C'est le point de départ de la construction de son

\footnotetext{
${ }^{22} \mathrm{Au}$ Brésil à l'époque mentionnée par Sylvia l'équivalent du Bac s'appelle vestibular et il est organisé par chaque université ; en général les enfants membres de la classe moyenne s'inscrivent dans un cours pre-vestibular pour se préparer aux examens de sélection.

${ }^{23}$ Taylor (2007: 401-423) transcrit une interview avec Mestre Sylvia dans laquelle elle explique clairement les problèmes familiaux qui ont motivé son départ à Londres.

${ }^{24}$ Espace réunissant des salles de spectacles et de cours, dédié à la danse, très important et réputé sur la scène artistique de Londres. Aujourd'hui c'est Mestre Poncianinho du groupe de capoeira Cordão de Ouro qui y propose des cours. Selon Sylvia, le Brazilian Contemporary Art a dû fermer ses portes l'an dernier à
} 
réseau. Ensuite, une responsable de l'ILEA (Inner London Education Authority), relevant du domaine de la défense personnelle, l'a invitée à donner des cours dans un centre à Holborn. Ces mêmes élèves deviendront, plus tard, des professores et contramestres $^{25}$.

Dès l'établissement des cours réguliers en 1988, elle commence à penser à inviter Mestre Sombra à Londres. La venue du mestre constitue un moment important dans l'organisation des groupes hors du Brésil. C'est le moment d'organiser un stage et de montrer le fruit de son travail aux autres groupes et au mestre. Son premier stage a eu lieu en 1991 à Londres. Mestre Sombra de Santos et Mestre Beija-Flor, à l'époque déjà établis à Paris, furent invités. En même temps à Londres avait lieu un festival brésilien avec la présence de seize mestres du Brésil, ce qui explique pourquoi les organisateurs avaient décidé d'organiser l'évènement de Sylvia conjointement à celui des mestres brésiliens ${ }^{26}$. Sylvia confie s'être sentie mise à l'écart à cause de la présence des autres mestres. C'était sa première expérience dans la coordination d'une rencontre et elle a bien ressenti le poids de la pression des mestres du Brésil.

Par la suite, elle organisera de nombreux stages et ajoute que Mestre Sombra revient chaque année. En 1991, elle a ouvert à Highbury le premier espace européen exclusivement consacré à la pratique de la capoeira, et c'est à ce moment précis qu'elle a fait venir Mestre Marcos, son futur mari, pour venir en aide à son groupe ${ }^{27}$. C'est grâce au soutien du réseau des groupes de capoeira - qui s'est, selon elle, organisé de façon spontanée - qu'a été possible l'arrivée de Marcos à Londres. Après s'être vu refuser l'entrée sur le territoire anglais, Marcos a décidé de ne pas retourner au Brésil. Il a débarqué au Portugal et y est resté pendant trois mois avec l'aide du groupe União de Mestre Umoi. Ensuite s'est présentée une possibilité de travail en Espagne et peu de temps après s'être installé en Espagne, il a rejoint la France, puis l'Allemagne. C'est donc en 1992, soit un an après son arrivée en Europe, que le groupe a réussi à obtenir son permis de travail au Royaume-Uni.

\footnotetext{
cause de la crise.

${ }^{25}$ Contra-mestre Agnes Folkestad, contra-mestre Gerard Taylor, professor Lagartixa - Antony Brian Early.

${ }^{26}$ C'est pour participer à ce festival que Rilene et sa sœur, les futures fondatrices du groupe Amazonas, sont venues du Brésil.

${ }^{27}$ District londonien d'Islington, Londres, Royaume-Uni.
} 


\section{La construction du genre dans le marché de la capoeira à Londres}

Le genre est socialement construit et les rapports entre «masculinité » et «féminité » sont donc contextuels. Dans les groupes de capoeira hors du Brésil, il est important de comprendre les variations entre les représentations «brésiliennes » de ce qu'est le «masculin» ou le «féminin» et les représentations locales de la «masculinité » et «féminité ». Butler (1988) affirme que le genre n'est pas inscrit passivement dans les corps et non plus déterminé par les facteurs biologiques, comme la nature, le langage ou des facteurs d'ordre symbolique. Ainsi, le « gendered body » joue dans ces contextes spécifiques et réalise des interprétations face aux directives préexistantes (Butler, 1988: 531).

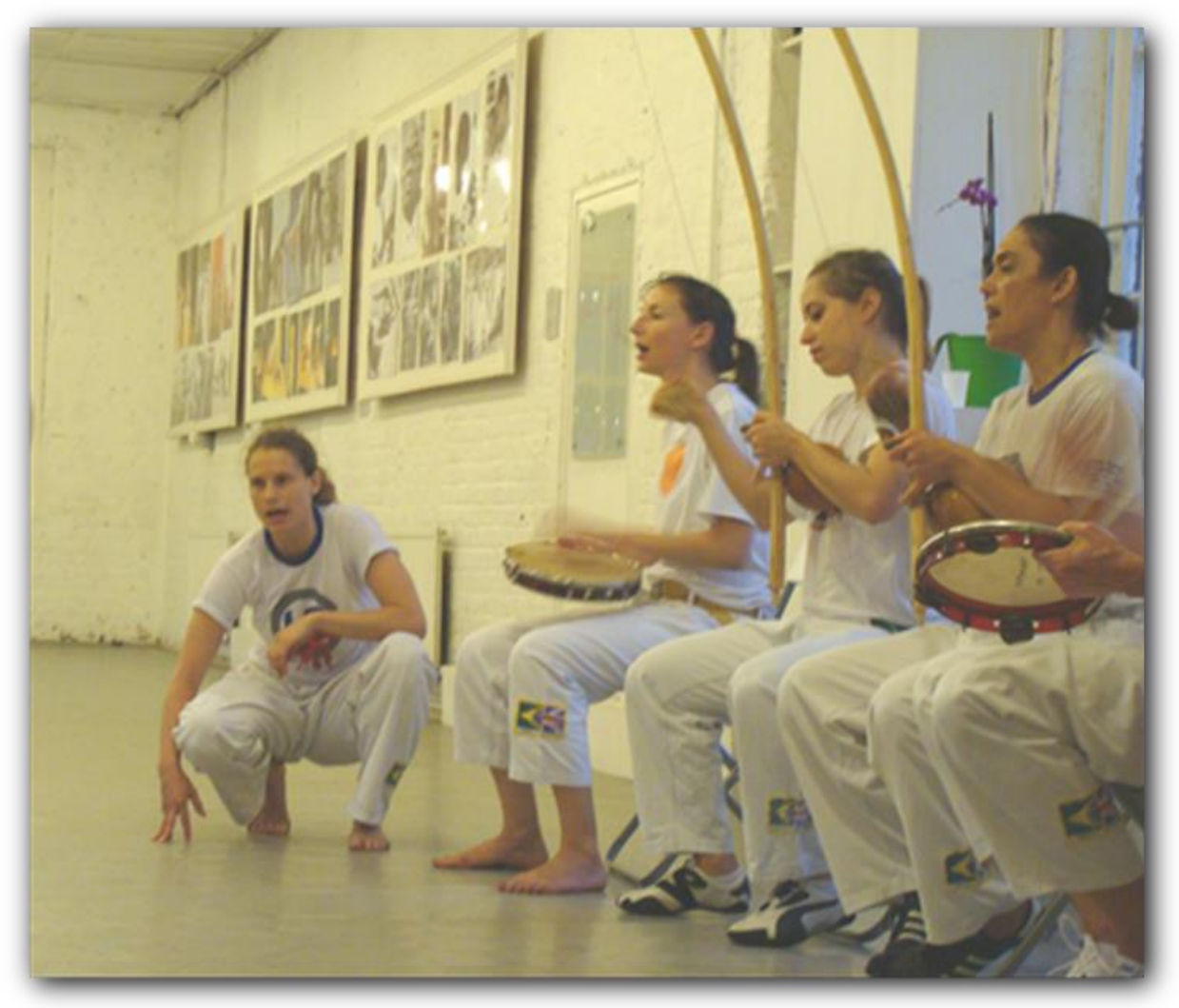

Figure 6: Roda de capoeira LSC $@$ Daniel Granada ${ }^{28}$

\footnotetext{
${ }^{28}$ Roda de capoeira dirigée par Mestre Sylvia au berimbau à droite, en arrière-plan apparaissent trois grands panneaux qui rassemblent les images des mestres de capoeira.
} 
Pour Butler (1990: 3) le genre n'est pas construit de manière cohérente et consistante selon les différents contextes historiques. Au contraire, il sera variable selon les intersections entre le genre et en fonction des facteurs comme la « race », la classe, l'ethnie et d'autres intersections qui peuvent exister avec des modalités régionales ou des identités constituées par les discours. Il est, de cette manière, impossible de dissocier le genre des rapports politiques et culturels au sein desquels il est contruit et maintenu: «As a shifting and contextual phenomenon, gender does not determine a substantive being, but a relative point of convergence among culturally and historically sets of relations »(Idem: 11) ${ }^{29}$. La délocalisation de la pratique au Brésil et sa rélocalisation à Londres, ainsi que les traductions faites par une mestre brésilienne au sein d'une pratique dont le marché est historiquement sous les auspices de la domination masculine, posent d'autres défis pour l'analyse et l'interprétation. Ces éléments trouvent leur place et sont renégociés dans l'organisation des groupes qui constituent le marché de la capoeira à Londres. Ils ont des répercussions sur l'organisation interne des groupes de capoeira, dans la construction des rapports entre hommes et femmes et s'expriment aussi lors des « jeux » de capoeira au moment des rodas. Ainsi, les caractéristiques des jeux sont elles aussi perméables aux représentations du genre partagées au sein des groupes de capoeira. Sur le terrain, j'ai pu entendre des commentaires comme « il joue comme une femme » de la part de capoeiristas se référant à un joueur masculin jouant avec peu d'efficacité sans faire de coups portés ou de rasteiras de forme effective, ou aussi pour faire référence à celui qui tombe facilement dans les pièges de l'autre. À contrario, d'une femme dont le «jeu » est plus agressif et dangereux pour son adversaire, certains capoeiristas diront « qu'elle joue comme un homme ». Le corps biologique est utilisé de la même manière pour expliquer la construction du genre dans les groupes. Il est fréquent d'entendre des femmes élèves affirmant sentir la limite de leur «corps de femme »dans certains mouvements, principalement ceux d'équilibre comme la bananeira alors que les hommes seraient plus aptes à réaliser des mouvements sollicitant la force musculaire ${ }^{30}$.

\footnotetext{
${ }^{29}$ Trad.: «En tant que phénomène mouvant et contextuel, le genre ne détermine pas un être substantiel, mais un point relatif de convergence entre un ensemble de relations culturellement et historiquement définies ».

${ }^{30}$ La bananeira est un mouvement de la capoeira qui consiste à se tenir en équilibre sur les mains.
} 
L'environnement masculin prédominant de la capoeira rend l'expérience de la femme mestre singulière à Londres:

\begin{abstract}
« Je n'ai jamais eu de difficultés à être femme et mestre de capoeira. Quand je suis invitée à un stage ils disent qu'ils m'invitent car je suis une femme. Ce que je ressens c'est que mes priorités ont changé. Ma priorité n'est plus la capoeira, ma priorité c'est ma famille et mes enfants et en deuxième plan peut être la capoeira. Je ne sais pas comment pensent les hommes, mais ce qu'ils pensent de moi ne m'inquiète pas, car je fais mon travail et j'essaie d'être présente dans la mesure du possible, car j'ai un compromis avec ma famille. Il est évident que les choses changent une fois que tu as des enfants ».
\end{abstract}

Ce passage met en évidence les questions liées au fait d'être une femme mestre de capoeira. Sylvia regrette que ses invités lors des stages lui demandent une implication inconditionnelle en dépit des grands changements engendrés par l'arrivée de ses enfants dans sa vie. Désormais, elle a une famille de quatre personnes. N'ayant personne à proximité pour l'aider avec ses enfants, il lui est devenu compliqué de participer aux évènements qui sont une charge en plus du travail de l'école de capoeira.

«Je ne peux pas faire les mêmes choses que d'autres mestres hommes, voyager tout le temps et participer aux stages car je dois m'occuper de ma famille. Donc il y a ce côté qui est différent car l'homme a plus de disponibilité pour être présent. Je ne connais pas beaucoup de femmes dans ma position. Je connais des femmes capoeiristas qui n'ont ni enfant ni famille ».

L'expérience de femme et de mère de Mestre Sylvia est centrale dans son vécu avec la capoeira, à la différence de celle des capoeiristas hommes, comme elle insiste à le souligner. L'autre question importante soulevée par Sylvia est que le fait d'être une femme a aussi des conséquences lors des stages et des «jeux» de capoeira. Elle doit penser à la façon dont elle va «jouer» lors d'une roda de capoeira afin de ne pas froisser son partenaire, s'il est un homme, lorsqu'elle arrive à le déstabiliser lors d'un coup porté ou d'une rasteira. Dans ce cas, elle doit savoir gérer et mesurer les limites du jeu.

D’autres femmes élèves interrogées rapportent des cas de partenaires de « jeu » hommes qui pendant un «jeu» de roda de capoeira peuvent se sentir humiliés de recevoir un coup d'une femme et réagissent différemment que si le coup venait d'un homme. 
L'autre point important dans l'établissement des rapports entre hommes et femmes dans le groupe réside dans les rapports de séduction; ceux des pratiquants nonbrésiliens envers les Brésiliennes et ceux des Brésiliens envers les pratiquantes nonbrésiliennes. Ces rapports sont établis sur des représentations du Brésil et des Brésiliens associées à la sensualité et à des mœurs considérées comme détendues et légères. Comme les groupes deviennent souvent des groupes d'amis, les relations affectives et les situations de séduction dans les groupes ne sont pas rares. Dans le cas de Mestre Sylvia, son statut respectable de mestre réduit considérablement ces situations. Elle affirme ne pas avoir été victime de préjugés ou de problèmes majeurs liés à des situations de séductions avec les pratiquants de capoeira masculins:

\begin{abstract}
« Je n'ai jamais eu de problèmes avec les hommes. Je pense que c'est grâce à ma posture. Les hommes qui veulent de la séduction ne viennent pas ici à l'école. Les groupes qui pensent de cette façon ne m'invitent pas. Car peut-être qu'ils ne valorisent pas mon travail et tu commences donc à circuler dans un cercle de personnes qui pensent différemment ».
\end{abstract}

Même si l'environnement de la capoeira a été pendant longtemps « masculin », et continue dans certains cas à être machiste, le parcours de Sylvia et la manière dont elle a conquis son espace au sein de cet environnement, montrent que les femmes y acquièrent de plus en plus de place. Dans les groupes que j'ai fréquentés à Londres, les femmes exercent une présence forte, pas seulement lors de l'entraînement, mais aussi dans l'organisation du groupe et des évènements. Fréquemment, ce sont elles qui prennent en charge la partie administrative des groupes et jouent un rôle primordial dans l'organisation des stages au sein des groupes.

La condition de mestre de capoeira reconnue par Mestre Sombra et son groupe au Brésil rend l'expérience de Sylvia singulière dans le sens où elle n'a pas le même statut que les autres femmes pratiquant la capoeira. Fréquemment dans les groupes, les élèves femme se plaignent de ne pas avoir accès aux berimbaus lors des rodas et affirment que les hommes ne les laissent pas chanter. Problèmes qu'en raison de sa position, Sylvia ne connait pas, comme l'atteste la définition du mestre de capoeira encadrée et affichée dans l'école $(c f \text {. Figure } 7)^{31}$.

\footnotetext{
${ }^{31}$ Il est à noter l'utilisation du féminin pour se référer au mestre de capoeira.
} 


\section{Le rapport entre les Brésiliens et les « étrangers »}

La difficulté vécue par les capoeiristas brésiliens qui arrivent en Europe pensant avoir trouvé la terre promise est bien expliquée par Sylvia. Elle insiste sur la précarité des débuts de ces aventuriers en Europe. Selon Sylvia, le marché étant de plus en plus saturé d'enseignants de capoeira, il devient réellement difficile pour les nouveaux venus de s'installer et de se faire accepter dans le «groupe de capoeiristas hors du Brésil ». Ce marché concurrentiel compte de nombreux pratiquants non-brésiliens de longue date qui réclament eux aussi leur place de porteurs légitimes de cette pratique.

Sylvia explique qu'il existe le mouvement des Brésiliens venus pour s'installer en Europe et le mouvement des autres qui viennent et qui repartent, mais qui souhaitent néanmoins maintenir un groupe sur place. Ces derniers désignent un non-Brésilien pour prendre en charge le groupe de capoeira pour assurer les cours à leur place. Ce qui, selon Sylvia constitue un avantage pour ces groupes qui préfèrent ne pas avoir de mestre sur place, ce qu'elle explique par une volonté d'absence de compromis avec la capoeira. Ils organisent des rencontres, cependant ne veulent pas de mestres et ne souhaitent pas avoir à en respecter un. Selon Sylvia, ce sont les mestres eux-mêmes qui commencent à perdre de la valeur, car il existe une prolifération de groupes formés par des personnes qui viennent donner des cours pour ensuite rentrer au Brésil. Cependant, ces personnes ne sont pas là pour orienter et diriger le travail du groupe au quotidien. Cette façon de faire, affirme Sylvia, rend possible la création d'autres idées sur ce qu'est la pratique de la capoeira, en raison d'un manque d'orientation appropriée dans leur apprentissage. Elle oscille entre l'envie de critiquer ce type de pratique et de valider cette forme d'appropriation de la capoeira. Selon elle, la capoeira est quelque chose de très «organique » dans son développement ${ }^{32}$. Il existe de nombreux nouveaux arrangements en termes d'enseignement et d'apprentissage de la capoeira, qui surgissent de la rencontre entre les Brésiliens et les non-Brésiliens hors du Brésil. De par sa position de mestre de capoeira, elle comprend qu'il est nécessaire de maintenir son statut et réprouve l'existence des expériences des pratiquants qui mettent en cause l'importance, ou même l'adhésion à un mestre dans le cadre de l'apprentissage de la

\footnotetext{
${ }^{32}$ Le mot « organique » au Royaume-Uni est utilisée pour identifier des produits sans engrais chimiques dont l'équivalent en français serait «biologique », mais sur le terrain, je l'ai entendu être fréquemment associé à un style de vie, ou à des pratiques qui seraient vues comme " positives », «pures » ou libres d' influences néfastes. Dans ce sens la capoeira, dans son développement, est fréquemment pensée comme « organique » par ses pratiquants.
} 
capoeira. Pour Sylvia, les formes d'enseignement de la capoeira qui ne respectent pas la forme «traditionnelle » d'organisation, sont regardées avec une sorte de mépris et de dégout. C'est comme si ceux qui pratiquaient la capoeira sans la présence ou l'orientation d'un mestre ne savaient pas exactement ce qu'ils faisaient, ou encore, comme s'ils faisaient quelque chose de différent de ce que l'on fait au Brésil, voire quelque chose d'autre que la capoeira.

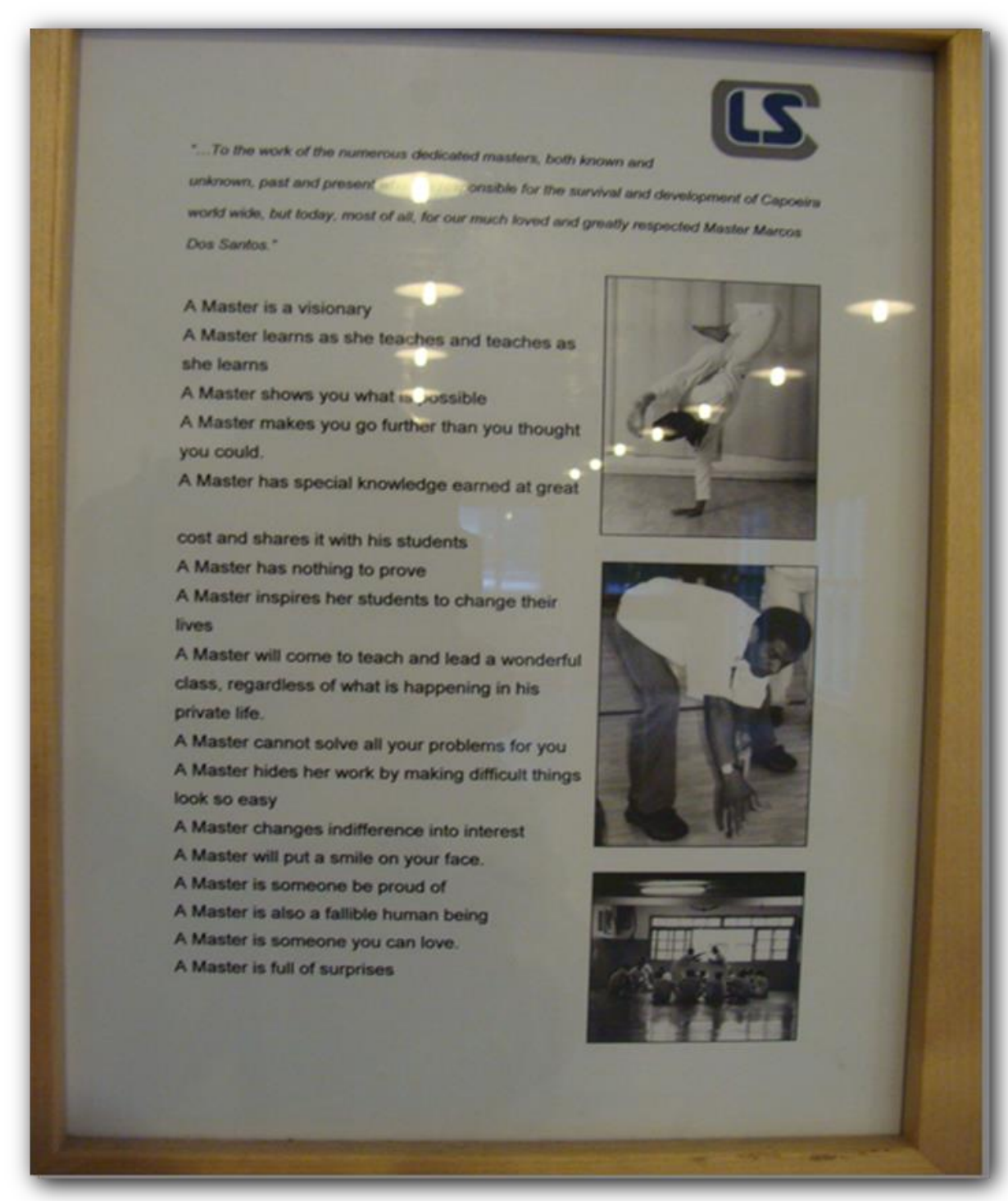

Figure 7: Cadre dans la salle de cours LSC $\odot$ Daniel Granada 
Le contrôle de la capoeira hors du Brésil, mis en place par les capoeiristas et principalement les mestres est basé sur la reconnaissance du statut du pratiquant en tant que pratiquant légitime de capoeira. Ce statut est prouvé grâce à la liaison du pratiquant à un mestre ou groupe reconnu et censé être légitime. Sylvia dans sa position de mestre de capoeira n'approuve pas ceux qui veulent apprendre la capoeira sans un mestre. Ce sont les mestres de capoeira eux-mêmes qui ont mis en place ce système de reconnaissance et qui le contrôlent, elle comprend donc son rôle dans le renforcement et la manutention de ce système basé sur la reconnaissance. Les groupes obéissant à la règle d'affiliation à un groupe qui possède un mestre légitime et reconnu seraient, d'après Sylvia, ceux qui respectent la forme «traditionnelle»d'organisation des groupes au Brésil. Pour les groupes de capoeira, la «tradition » est un critère utilisé pour classer les « légitimes » et les «non légitimes » au sein de ce marché. La légitimité doit être acquise à condition d'être sous les auspices d'un mestre légitime.

\section{Le retour au Brésil}

Sylvia ressent l'importance croissante du Brésil sur la scène économique et culturelle mondiale comme un des facteurs expliquant l'augmentation des groupes de capoeira hors du Brésil. Elle observe en Europe, et particulièrement à Londres une certaine curiosité envers la « culture brésilienne ». Par ailleurs, Sylvia explique qu'elle ne parvient pas à avoir une vision de ce que deviendra la capoeira à Londres d'ici dix ans. Elle remarque que les capoeiristas brésiliens sont en train de rentrer au Brésil avec la crise économique qui s'est installée en Europe depuis 2008 et que plusieurs rapportent dans leurs bagages les leçons apprises à l'étranger: il s'agit de l'expérience par rapport à l'organisation des groupes de capoeira et d'un peu d'argent économisé. Toutefois, si Sylvia confie que d'autres personnes résidant à l'étranger depuis longtemps ressentent une certaine lassitude en travaillant avec la capoeira. Par contre, d'autres capoeiristas avec qui j'ai pu faire des entretiens ont exprimé une grande motivation et l'envie de continuer leur travail hors du Brésil.

Sylvia se montre préoccupée par l'avenir de la capoeira en Europe et se demande où la nouvelle génération des capoeiristas à l'étranger va amener la capoeira, parce qu'il existe plusieurs capoeiristas qui pratiquent la capoeira de formes diverses et variées, en proposant par exemple des formules de cours de formation rapide, ou en 
devenant « coach particulier » de capoeira. Sylvia s'oppose à ces nouvelles formes de cours de capoeira et réaffirme l'importance du mestre et du groupe dans l'apprentissage.

Si elle réfléchit depuis dix ans à rentrer, elle a conscience que la vie est différente au Brésil. Là-bas, elle pense qu'il faudrait au moins une dizaine d'années pour que son travail et la manière dont elle et son mari organisent leur groupe soient reconnus. Pour l'instant, elle n'est pas en mesure de pouvoir se permettre de perdre ce temps-là. 


\section{La LSC Herança}

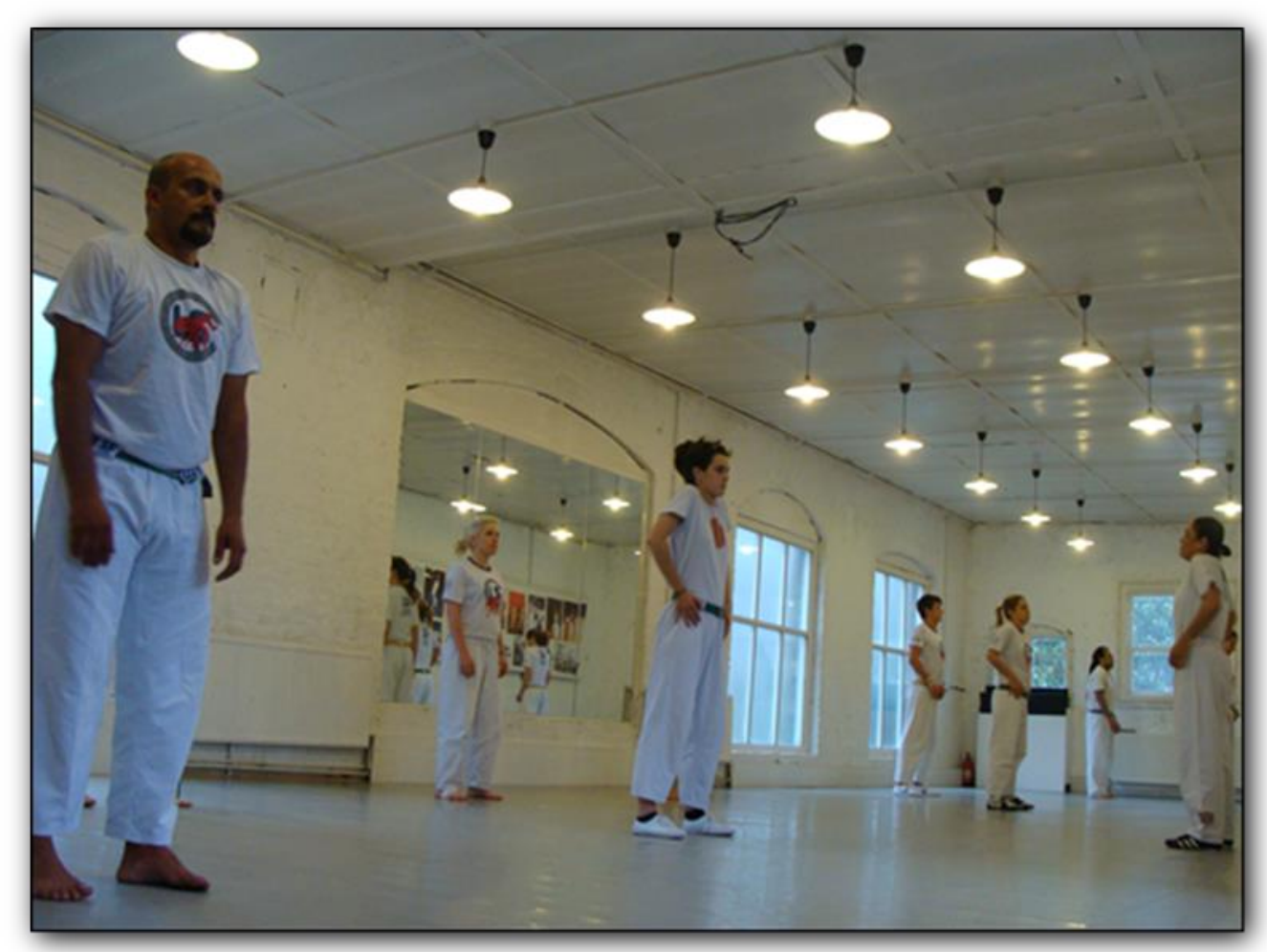

Figure 8: Entraînement LSC @ C Daniel Granada

Le groupe de capoeira à Londres comptait, en 2011, environ une trentaine d'élèves. Selon Sylvia, avec un tel effectif, il devient difficile de maintenir l'espace. En effet, la faible fréquentation serait due à la crise économique mais aussi à la concurrence sur le marché d'autres groupes dont les cours sont moins onéreux qu'à la LSC. Le groupe de Sylvia organise ses batizados une fois par an, mais même cet évènement représentatif pour le groupe se voit perdre de l'importance. Le festival de 2004 était complet, elle avait loué un grand théâtre de 600 places à la City pendant deux soirées, alors que, l'année dernière, il s'agissait de 150 places pour une seule soirée. D'après Sylvia, cette baisse de fréquentation s'expliquerait par la présence d'autres groupes sur le marché et la banalisation des spectacles de capoeira à Londres. Pour elle, les responsables de la dévalorisation la capoeira sont les groupes qui organisent des spectacles de mauvaise qualité. 
Leur groupe ne fréquente pas d'autres groupes en dehors de ceux appartenant au réseau du Senzala de Santos. Cette caractéristique a engendré une certaine méfiance de la part des autres groupes qui pensent que la LSC les snobe en écartant tout contact avec les autres. Eviter les rapports avec les autres groupes peut être vu comme une stratégie consistant à marquer sa différence et sa singularité face à ceux qui se sont installés par la suite. Cela peut être vu comme une manière de marquer une différence au niveau de la capoeira qu'ils pratiquent. Sylvia explique toutefois cette distance par un agenda très chargé en raison des nombreuses activités réalisées dans l'espace du LSC et le souhait de consacrer du temps à sa famille. Elle admet ne pas être disponible pour rendre visite aux autres groupes de Londres et ajoute que sa participation aux stages des groupes proches s'est réduite à la suite de la naissance de ses enfants.

\section{Une école, un business}

Quand on fréquente l'espace de la LSC, lors des jours d'entraînement, on remarque que la préoccupation de l'organisation tourne autour de la propreté de l'espace, l'uniformisation des élèves impeccables dans leurs tenues blanches, puis du contrôle du temps et de l'organisation de la routine de l'entraînement ${ }^{33}$. On a l'impression que rien n'est laissé au hasard. Un autre exemple de la distinction de leur travail est l'adoption de la ceinture. À la différence d'autres groupes au Brésil ou ailleurs qui utilisent une corde nouée à la ceinture comme signe d'ancienneté et du niveau acquis au sein de la capoeira, Marcos et Sylvia ont décidé d'adopter la ceinture qui, selon eux, donne un côté plus organisé que la corde «qui bouge dans tous les sens ». L'utilisation des uniformes de l'école pour les entraînements physiques et pour les rodas est prise en compte très sérieusement par tous. Les pantalons blancs de l'école sont attachés à la taille grâce aux ceintures adoptées, les différentes couleurs désignent le grade de l'apprenant au sein du groupe ${ }^{34}$.

\footnotetext{
${ }^{33}$ Les cours commencent et se terminent à une heure précise au Royaume-Uni. Il n'est pas rare dans les groupes que j'ai visité au Brésil de voir les entraînements commencer en retard et dépasser l'heure de fin prévue, ceci même lors des stages.

${ }^{34}$ Les couleurs de ceintures dans la LSC sont des combinaisons de vert, bleu, jaune et blanc. Ces couleurs et leurs associations désignent l'ancienneté au sein du groupe et le grade dans la capoeira. Il existe 15 grades : cinq pour les enfants et dix pour les adultes, à chaque batizado certains élèves progressent selon l'avis des mestres.
} 


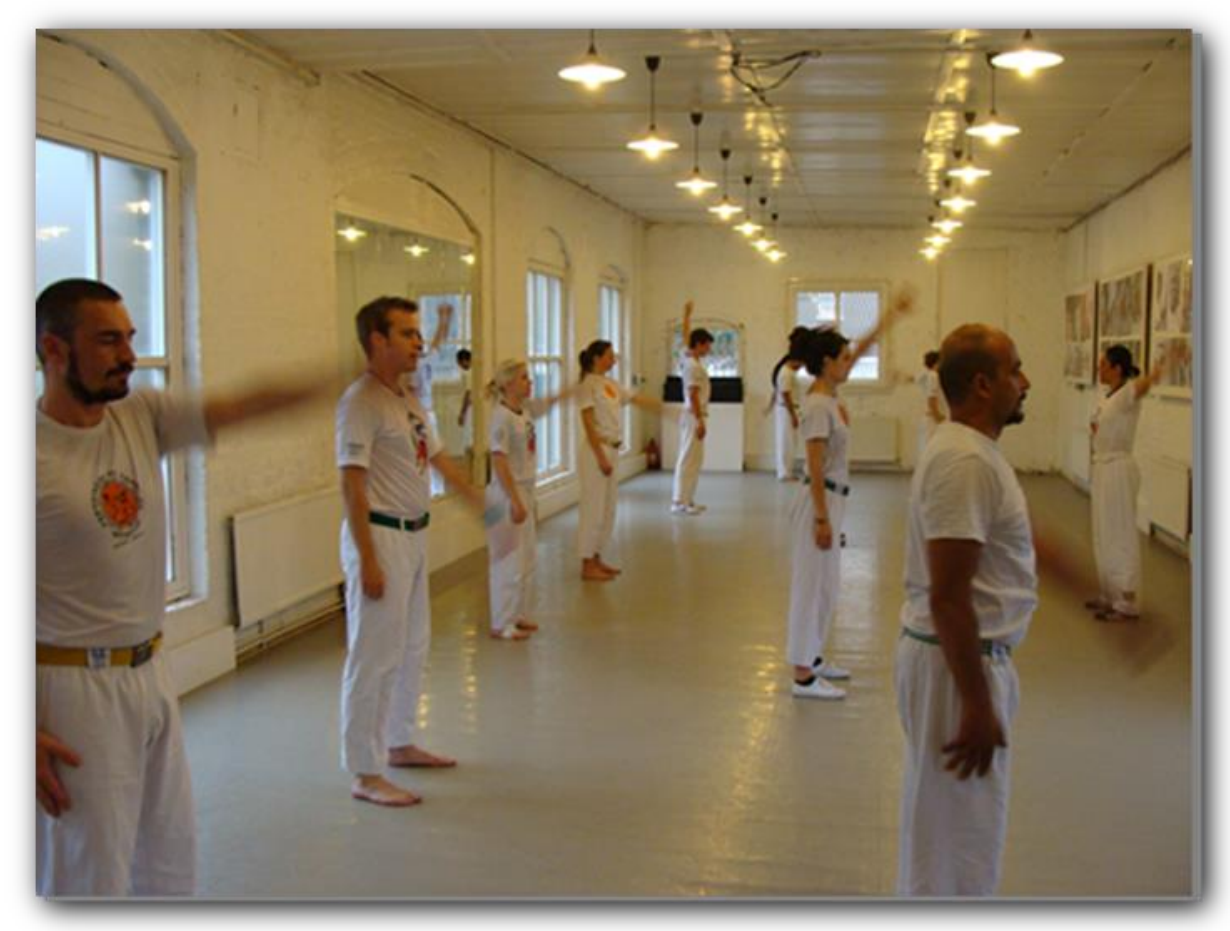

Figure 9: Échauffement LSC @ Daniel Granada

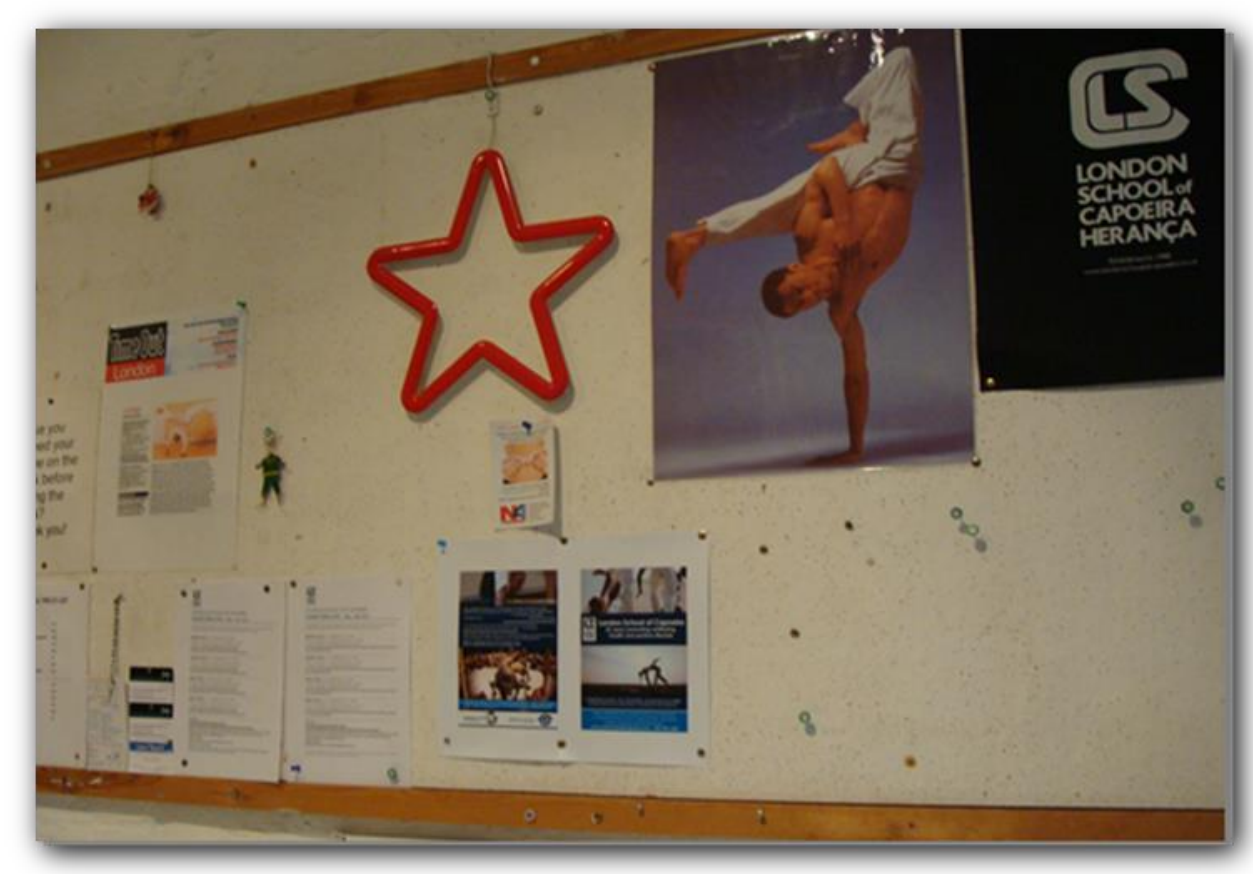

Figure 10: Panneau d'affichage LSC C Daniel Granada ${ }^{35}$

\footnotetext{
${ }^{35}$ Les paroles des chansons de capoeira avec leur traduction en anglais figurent en bas à gauche du tableau d'affichage.
} 
Pour une personne habituée à l'organisation des espaces d'entrainement de capoeira au Brésil, l'espace de LSC est tout de suite perçu comme singulier. Il est évident que la préoccupation des Mestres Sylvia et Marcos est d'offrir un service inspiré des écoles de danse et de théâtre de Londres. L'élève se trouve face à une infrastructure: sur les tableaux se trouvent les images des mestres, les paroles des chansons en portugais sont traduites en anglais et disponibles à la consultation sur le mur de l'école, les instruments sont accessibles et en bon état. Une préoccupation de professionnalisme s'exprime par un degré remarquable de formalisation et se traduit dans l'institution des règles et des codes de conduites qui régissent l'organisation du groupe à l'instar des écoles des autres modalités de danse à Londres, avec le maintien d'une distance entre mestre et élèves dans le cas de LSC.

L'axiomatisation de la pratique de la capoeira est perceptible dès le premier contact avec la LSC. Dans mon cas, ce contact s'est effectué par courriel. Le fait de savoir automatiquement que sous $24 \mathrm{~h}$, j'aurais une réponse à mon enquête, puis d'avoir été ensuite contacté par une tierce personne qui m'a fourni le téléphone de Mestre Sylvia pour convenir d'un rendez-vous, m'a montré une démarche très différente. En général, c'est le mestre ou le professeur lui-même qui répond aux messages. D’autres facteurs corroborent ces arguments à propos du formalisme plus remarquable au sein de ce groupe, comme la possibilité d'acheter en ligne des forfaits pour les entraînements, des instruments, livres, CDs, DVD du groupe. La LSC loue également ses salles pour des activités diverses selon les disponibilités de l'agenda de l'école. Dans les rapports personnels entre les membres du groupe, cette formalisation se montre au niveau des prestations de service aux élèves, qui sont perçus comme des clients. Cette démarche contraste avec le haut degré d'informalité qui peut exister dans certains groupes principalement au Brésil, mais aussi à l'étranger. Les mestres de capoeira ont tendance à personnaliser les rapports de domination à l'intérieur des groupes à un tel degré qu'ils mélangent les liaisons professionnelles et les rapports personnels, exerçant un certain paternalisme et effaçant la distance à laquelle les élèves européens sont habitués.

La posture de Mestre Sylvia lors des entraînements est sérieuse et marque une distanciation envers ses élèves, l'aura de respect et même une certaine révérence est palpable. Ses élèves connaissent bien la routine des entraînements, il n'y a pas de place pour l'improvisation car le système est établi depuis longtemps. 
Cette organisation laisse transparaître l'adaptation frappante à une façon de faire qui peut être vue comme «britannique ». Durant l'entrevue, Sylvia y fait référence comme un exemple d'organisation à suivre. C'est elle-même qui a mis en place l'organisation de la LSC. Elle est en grande partie responsable des exigences liées à l'image du groupe et à son organisation si différente de la forme dont la capoeira était pratiquée au Brésil. Mestre Marcos explique:

\begin{abstract}
«Si pour certaines écoles de capoeira, cette forme d'organisation est nouvelle, pour nous cela est déjà vieux. Quand je suis arrivé ici, cela existait déjà. C'est Sylvia, Mestre Sylvia la responsable de cette organisation. C'est à mon arrivée que je me suis dit: - Ça alors! Tout est bien organisé ici ! Et j'ai commencé à comprendre ce système. Je ne suis pas venu ici en étant déjà au courant de ça. Non, j'étais naïf à ce sujet. Je donnais des cours de capoeira au Brésil. Mais je ne savais pas faire de l'argent avec la capoeira. C'est ici, quand j'ai commencé à parler avec les personnes, quand j'ai commencé à me mettre en valeur, que j'ai appris et je suis encore en train d'apprendre et je n'ai pas peur de parler de cela ».
\end{abstract}

Le témoignage de Mestre Marcos prouve que sa femme a imposé un niveau d'organisation très poussé et que Marcos ignorait pendant son expérience de professeur de capoeira au Brésil. Il est important de noter la capacité d'action individuelle, dans ce cas, l'initiative de Sylvia en tant qu'organisatrice d'une forme de pratique de la capoeira différente de celle qu'elle a connue au Brésil. Sans aucun doute, en prenant en compte le contexte local, elle a senti la nécessité d'imposer de nouvelles caractéristiques à la pratique de la capoeira pour être acceptée par le public londonien et devenir de cette façon, plus rentable. Certes, Mestre Marcos joue aussi un rôle important dans l'organisation et le fonctionnement de l'école de capoeira, mais il souligne lui-même l'importance de Sylvia, qui serait à l'origine de ces adaptations au contexte local. 


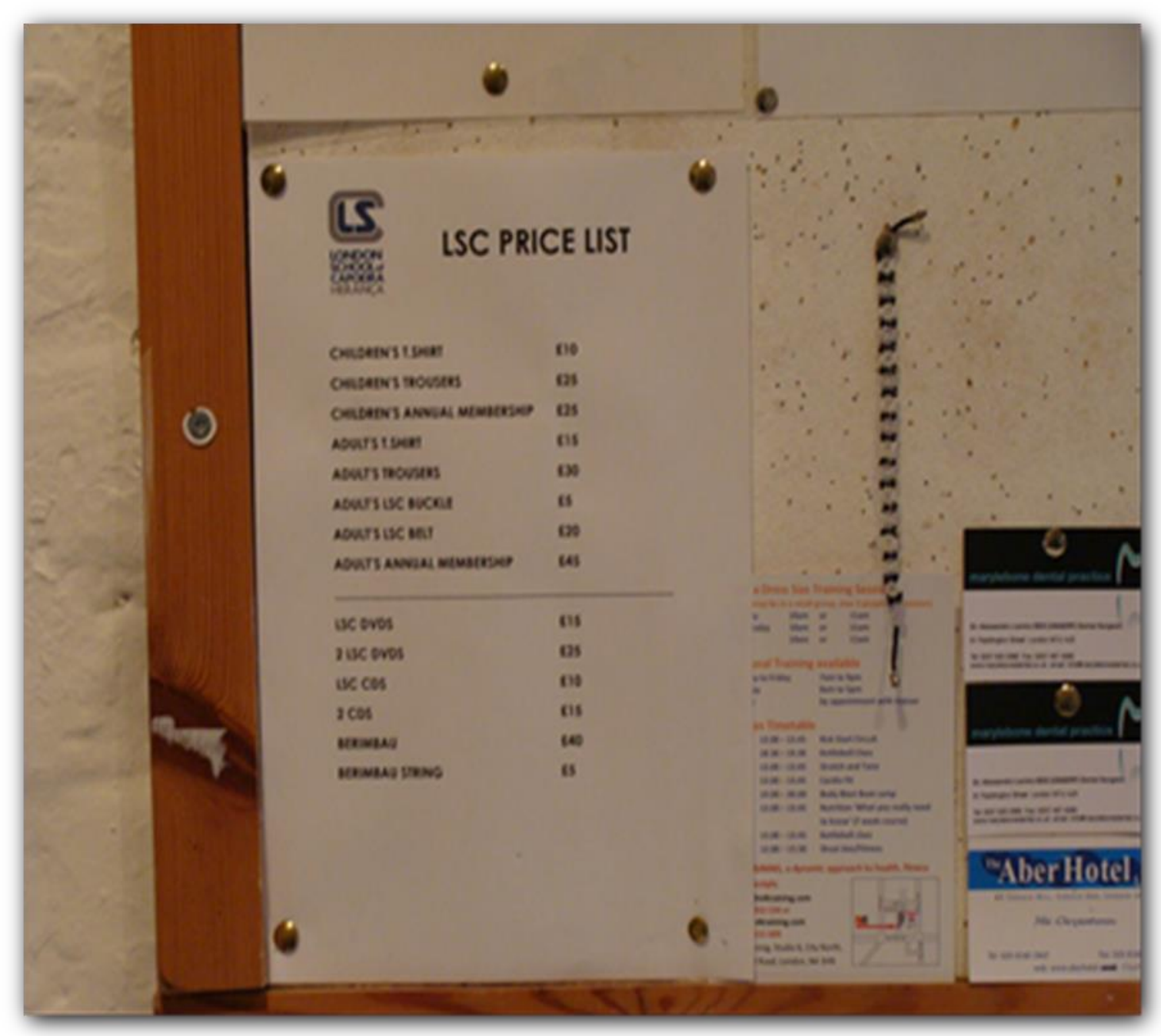

Figure 11: Liste des prix des produits LSC 2011 @ Daniel Granada

\section{La professionnalisation du mestre}

Avec la venue de Mestre Marcos, le couple a décidé de monter une compagnie de danse et de théâtre appelée Passo a Passo en 1993. Après avoir réalisé quelques représentations ensemble, Sylvia affirme s'être heurtée au manque de professionnalisation du mestre de capoeira:

« Nous nous sommes retrouvés face à un problème qui est celui du manque de professionnalisation du mestre de capoeira car tu sais qu'aujourd'hui le mestre de capoeira ne peut pas être qu'un capoeirista. Il doit aussi avoir une didactique, avoir un bon comportement. Il doit chanter et jouer des instruments. Il doit pouvoir transmettre son savoir aux élèves. Il doit savoir faire un virement bancaire. Il doit savoir mettre à jour son site internet puisque maintenant l'information n'est plus passée directement car la technologie d'une certaine manière a aidé et 
dérangé. On est obligé de faire des choses qu'on est pas censé faire mais il faut les faire ».

Le récit de Sylvia montre que le métier de mestre de capoeira est en train de changer, qu'il ne suffit pas de «jouer» et d'enseigner la capoeira mais qu'il faut répondre aux nouveaux besoins qui obligent les capoeiristas à adapter leur pratique. Le mestre doit savoir maitriser les technologies pour mettre en valeur sa pratique. Le mestre doit aussi avoir, d'après Sylvia, un comportement correct.

La professionnalisation du mestre de capoeira et sa spécialisation chaque fois plus nécessaire est une adaptation de la capoeira aux nouveaux contextes, une tendance qui s'était manifestée au Brésil, mais qui, avec l'expansion de la pratique, s'est potentialisée et a atteint de nouveaux domaines. Par exemple, la maitrise de la langue locale est un élément important. Les mestres de capoeira réussissant le mieux hors du Brésil sont ceux qui arrivent à comprendre et parler la langue, comprendre le contexte local, les besoins des élèves. Ils doivent adapter leur pratique. Avec l'expansion et la diversification des groupes, le mestre qui essaie de faire du profit rapidement avec la capoeira n'a plus sa place face aux mestres qui possèdent une vision à long terme et qui cherchent à développer leur groupe sur place.

Certes l'exemple de Sylvia et Marcos est pionnier dans le mode de direction de la LSC, mais les mestres qui cherchent à constituer un groupe et faire un travail respectueux envers leurs élèves sont de plus en plus nombreux. Les cas des mestres qui cherchent à faire du profit rapidement et dans certains cas, à tromper les élèves, sont devenus rares et ponctuels en Angleterre grâce au développement de la pratique et au contrôle opéré par leurs pairs hors du Brésil.

\section{LSC et le lien avec le Brésil}

La liaison entre le groupe à Londres et le groupe au Brésil est maintenue par le biais de l'apport financier. Le don d'argent ou son euphémisme, «l'aide » apportée au groupe de Santos, est ponctuel et accordé dans la mesure du possible d'après Sylvia. Une autre façon «d'aider » le groupe est de faire venir Mestre Sombra et ses élèves, ou d'autres mestres liés au groupe. Le groupe prend en charge les billets et le séjour des mestres qui reçoivent une somme d'argent en rémunération pour leur participation aux batizados et autres stages organisés par la LSC. Ce lien entre le groupe au Brésil et celui 
de Londres est central pour conférer de la légitimité au travail de Sylvia et Marcos face à d'autres groupes de capoeira présents sur le marché de Londres. C'est la liaison avec son mestre au Brésil et le fait de venir d'une école de capoeira brésilienne qui a permis à Sylvia de se lancer en tant que pionnière à la tête d'un travail de capoeira à Londres. La possibilité d'attester d'une liaison de descendance avec une école et un mestre censé être légitime est un facteur qui confère du pouvoir au pratiquant qui se lance en tant que professeur de capoeira hors du Brésil.

En complément à l'apport financier transformé en «aide », il existe aussi un échange qui se fait par l'envoi d'élèves du groupe de Londres au siège du groupe au Brésil. Cet échange n'est pas toujours sans problème en raison des adaptations de la pratique de la capoeira à la «culture locale». Selon Sylvia, la capoeira varie selon la région du Brésil et elle est donc encore plus différente lorsque pratiquée à l'étranger. En réponse à ma question sur la nature de ces adaptations, elle explique d'abord que, étant sur place, elle ne se rend plus bien compte des changements, puis cite le fait d'être plus directe. Selon elle, cette forme plus directe d'agir et de penser n'est pas bien comprise par les Brésiliens qui sont plus malléables et plus flexibles dans le traitement de certaines questions. Elle sent qu'elle est en train de perdre cette caractéristique au fur et à mesure qu'elle vit à l'étranger.

Elle affirme que les capoeiristas brésiliens vivant au Brésil ne comprennent pas leur façon de vivre à Londres, ils pensent qu'ils ont une vie facile et qu'ils se sont enrichi avec l'argent de la capoeira. Ces capoeiristas ne réalisent pas les difficultés qu'ils rencontrent pour faire leur travail et pour promouvoir une image positive de la capoeira et du Brésil. Selon Sylvia, «l'échange culturel » entre Brésiliens et nonBrésiliens peut être parfois vraiment compliqué, voire problématique:

\footnotetext{
«Les élèves qui partent de l'école pour aller au Brésil et qui n'ont jamais vu la capoeira en dehors de l'école et des vidéos sur internet sont habitués au niveau d'organisation de la LSC. Quand ils arrivent au Brésil, ils ne comprennent rien. Soit ils détestent. Soit ils adorent».
}

Sylvia conseille à ses élèves de ne rien changer à leur capoeira lors de leurs séjours au Brésil. Elle leur recommande de se comporter de la même façon qu'à la LSC: respecter les règles de bonnes manières, se présenter au mestre de capoeira du local des entraînements ou des rodas, demander le tarif du cours et le payer sans discuter. Sylvia remarque que dans certains cas, les élèves sont tellement excités qu'une fois au Brésil, il 
arrive qu'ils aient un comportement indésirable. Inspiré par les histoires racontées sur le Brésil et les Brésiliens, l'élève non-brésilien peut avoir un comportement stéréotypé, dans l'objectif de ne pas être vu comme un «gringo ». Il peut vouloir aller dans l'extrême opposé et se comporter en essayant d'imiter les «Brésiliens », par exemple en voulant un traitement de faveur, ce qui serait mal vu.

D'autre part, selon Sylvia, quand le capoeirista est plus expérimenté et a l'esprit ouvert, il peut arriver à se rendre compte qu'au Brésil les choses se passent différemment. Il peut comprendre la singularité de la façon dont les bases de la capoeira sont transmises à Londres. Soit l'élève accepte et est émerveillé, soit il se demande «Mon Dieu, c'est quoi ça ? » et ne veut plus pratiquer la capoeira parce qu'il se dit que ce n'est pas la même pratique à laquelle il a été initié. Mestre Sylvia comprend que les adaptations réalisées au Royaume-Uni sont tellement importantes qu'elle affirme que les élèves risquent de ne pas reconnaître la capoeira une fois sur place. Elle explique qu'il existe des différences entre la capoeira pratiquée dans la LSC et celle pratiquée au Brésil et que, peu importe l'effort mené à l'intérieur de l'école pour expliquer ces différences aux pratiquants britanniques, parfois ils se sentent choqués lors de leurs séjours, car ils ne s'attendent pas à ce qu'ils trouvent au Brésil. La rupture avec la forme de la pratique de la capoeira au Brésil est si profonde qu'elle-même avoue que l'élève peut ne pas s'identifier. Il peut même aller jusqu'à vouloir arrêter la capoeira après son séjour au Brésil. Le degré de formalisme instauré par Sylvia à l'organisation de la LSC est la référence qui peut amener l'élève à se sentir surpris lors de ses séjours au Brésil. En effet, le contraste avec l'informalité, le désarroi de certains groupes, les rodas, les rapports des mestres avec les élèves et l'organisation des groupes devient le motif central du désaccord entre ce qu'ils ont appris à Londres et ce qu'ils retrouvent au Brésil. L'axiomatisation de la capoeira opérée par Mestre Sylvia, avec l'adaptation profonde à la manière « anglaise » dans le processus de relocalisation de cette pratique à Londres est une véritable traduction de la pratique brésilienne au contexte local, où Sylvia est l'interprète et le traducteur entre les deux mondes. 


\section{L’avis des élèves}

\section{Cleo}

Ayant complété trois années de capoeira en 2011, Cleo, jeune Londonienne, a découvert la capoeira lors d'un séjour au Brésil. Elle rapporte qu'en rentrant à Londres, elle a cherché pendant plusieurs mois sur internet avant de trouver le site de la LSC. Elle raconte avoir déjà fréquenté d'autres écoles de capoeira, mais jamais les rodas des groupes extérieurs. Selon elle, ce qui l'attire dans la capoeira, c'est la combinaison entre le mouvement et la musique ajoutée à l'harmonie intérieure que la pratique de la capoeira lui apporte. La capoeira lui offre une autre expérience de prise de conscience du corps et de l'esprit.

C'est également la combinaison entre la musique et la danse qui est, pour elle, « très spéciale, unique et une révélation par rapport à tout ce qu'elle connaissait auparavant ». Elle apprécie l'ambiance de son école et affirme «s'y sentir bien ». Les facteurs principaux de son adhésion à la LSC sont la liaison de son école à un héritage dans la capoeira et le fait qu'elle ressente que «peu importe d'où viennent les gens, ce qu'ils font dans leurs vies personnelles ou même où ils vont aller après, tous sont les bienvenus ».

\section{Faisal}

Le capoeirista Faisal est un homme ayant une quarantaine d'années. Il pratique la capoeira depuis dix ans et est fier d'ajouter qu'il a appris tout ce qu'il sait de la capoeira dans son groupe à Londres. Pour lui, la capoeira est une pratique fascinante grâce à laquelle « on est toujours en train d'apprendre avec soi-même, avec les autres pratiquants du groupe et avec ses mestres ». Dans son récit, Faisal exprime une certaine révérence envers ses mestres et même après tant d'années passées dans le groupe, il emploie les termes Mestre Sylvia et Mestre Marcos pour marquer le respect et la distance envers ses professeurs. Le temps de pratique de la capoeira dans la même école est un facteur qui renforce son respect et admiration par rapport au savoir acquis. 
Il s'enorgueillit de parler un peu la langue portugaise, ce qu'il estime être nécessaire à partir d'un certain stade d'apprentissage, afin de mieux comprendre la «culture brésilienne» et d'approfondir ses connaissances sur la capoeira. Puisque, remarque Faisal: « la capoeira n'est pas que le mouvement mais l'histoire, la culture et la musique qu'il faut maitriser ». Cela explique aussi ses séjours répétés au Brésil dont il ne sait pas exactement préciser le nombre puisque ce dernier est entrepreneur et ses voyages ont, parfois eu d'autres objectifs que la capoeira. Faisal rapporte avoir été dans le Sud du Brésil pour faire fabriquer les tennis Jinga, marque qu'il a lui-même lancée en Europe $^{36}$. Il précise que le taux d'échange convenable permettait le paiement d'un prix juste au producteur et favorisait ainsi la pratique d'un commerce équitable.

Même s'il est formado, il ne fréquente les autres groupes et leurs rodas qu'en spectateur. Fier d'être londonien, son apprentissage s'est fait au sein du groupe. Il ajoute n'avoir eu aucun problème au Brésil et affirme s'être senti très à l'aise lors des rodas avec ce qu'il avait appris dans son école londonienne. Faisal aime la capoeira mais n'a pas comme objectif de devenir un jour mestre de capoeira. Son plaisir est de pratiquer la capoeira «tant que son corps lui permet encore de le faire».

\section{Nicky}

Nicky a commencé la capoeira à la LSC quatre ans avant notre entrevue. Pour cette Londonienne originaire du West Country, la capoeira est une bonne activité et une manière agréable de se maintenir en forme: «C'est bon pour la souplesse car ça fait travailler différents muscles du corps ». Elle explique qu'elle pratique la capoeira surtout pour garder la forme et la santé sans avoir réellement le souhait de progresser dans la capoeira : «Si je ne faisais pas de capoeira, je choisirais un autre sport ». La capoeira est son activité physique de prédilection et lui permet de rester en forme tout en s'amusant.

Si elle affirme que le capoeirista doit apprendre la langue et la musique, sa connaissance de la langue portugaise se limite néanmoins aux chansons. Pour Nicky, le groupe de capoeira est un bon groupe social où elle a des amis avec lesquels elle sort régulièrement. La jeune capoeirista n'a jamais fréquenté d'autres groupes en dehors de

\footnotetext{
${ }^{36}$ Ces chaussures pour capoeiristas destinées au marché européen ont été produites à Novo Hamburgo, ville de colonisation allemande du Sud du Brésil.
} 
la LSC et n'a jamais été au Brésil. Ce qu'elle connait de la capoeira se résume à ce qu'elle a appris dans son école.

Les profils des élèves de la LSC montrent le rapport endogène du groupe. L'apprentissage des élèves se restreint à ce qu'ils apprennent dans leur école de capoeira, en contraste avec d'autres groupes de capoeira de Londres. Il est possible de voir une manière de maintenir le contrôle sur les élèves et de marquer la différence entre « nous » et les « autres ». De cette façon l'identité collective de la LSC se forme à partir du contraste ou des frontières symboliques qui vont distinguer leur groupe des autres qui sont sur le même marché. Ce processus est semblable aux idées de Barth (1998) par rapport à l'appartenance ethnique. Ce processus de distinction des autres sert à ajouter de la valeur au groupe lui même et à ses membres.

\section{Considérations finales}

L'indéniable réussite de Sylvia, Marcos et leur groupe a, d'une certaine manière, creusé la distance qui les sépare du Brésil. Cette sensation est vécue par Sylvia avec une certaine nostalgie, même si sa réussite et celle de son école de capoeira sur le « marché des produits ethniques » de Londres a été rendue possible grâce à la valorisation positive de son «identité brésilienne ». L'adaptation au contexte local s'opère par le biais d'une organisation sous forme d'école de capoeira avec des règles strictes qui passent par le respect des horaires des cours, le port d'un uniforme, l'organisation et la propreté de l'espace, l'établissement d'un rapport de prestation de service et de client distinct du rapport entre le mestre et l'élève existant au Brésil, conséquence de la formalisation imposée par Sylvia. Le plus grand degré d'institutionnalisation de la pratique de la capoeira s'exprime au travers de stratégies de pénétration dans les médias, les reportages dans des journaux, les publicités et les spectacles de danse avec la capoeira. Cependant, Sylvia signale qu'elle ne sera jamais anglaise et que ses élèves ne pourront jamais être brésiliens. Cette question n'empêche pas qu'ils deviennent d'excellents capoeiristas, qu'ils apprennent la langue et la « culture brésilienne », qu'ils maîtrisent les chants, les musiques et les instruments de la capoeira.

Selon Sylvia les personnes qui rejoignent son groupe sont des personnes qui s'identifient avec la façon dont elle conduit son travail, qui cherchent une capoeira plus organisée, un endroit pour passer un bon moment et participer à des activités 
intéressantes. Les personnes recherchent un profil du groupe de capoeira où l'idiosyncrasie du mestre de capoeira et sa relecture de la pratique constituent les grands responsables de la création de cet univers.

Il devient clair que pour Sylvia l'appartenance au groupe se fait par le biais de l'identification et l'adhésion à la façon dont elle travaille et à sa forme particulière de présenter la capoeira au Royaume-Uni. Cette adéquation au contexte local passe par sa capacité à adapter une pratique brésilienne au public de Londres due en partie à son habitus et de l'autre côté à sa compréhension du contexte local. Les modifications imposées à la capoeira par Sylvia vont dans le sens de la transformer en une pratique «organisée » selon les termes des écoles de danse de Londres. Elle est la grande responsable du formalisme présent dans son groupe de capoeira sur la scène de la capitale londonienne.

Ce qui ressort de l'étude du récit de vie de Sylvia et la création de son école de capoeira à Londres c'est que l'insertion dans le processus de transnationalisation favorise l'augmentation du capital symbolique (Bourdieu, 2001: 210) dans les deux pôles du circuit. Le fait d'être venu à Londres, associé à ses compétences en tant que danseuse et capoeirista est probablement ce qui a rendu possible le fait qu'une femme s'impose dans ce marché. Dans ce sens la valorisation positive d'un «bien culturel » du pays d'origine opère comme une stratégie d'insertion valable, qui permet l'intégration dans la société d'accueil. En adaptant la capoeira aux pratiquants locaux Sylvia opère des modifications conséquentes avec la forme selon laquelle la capoeira était pratiquée au Brésil, ce qui fait d'elle une interprète capable de modeler la pratique brésilienne dans une pratique transnationale.

\section{Référence}

ACETI, Monica. 2011. Devenir et rester capoeiriste en Europe. Transmissions interculturelles et mondialité de la capoeira Afro-Brésilienne. PhD Dissertation. Université de Franche Comté. ASSUNÇÃO, Matthias Röhrig. 2005. Capoeira: The History of an Afro-Brazilian Martial Art. London: Routledge.

BARBOSA, Maria José Somerlate. A Mulher na Capoeira. Arizona Journal of Hispanic Cultural Studies, 2005, vol.9, p. 9-28.

BARTH, F. (1998), "Grupos Étnicos e suas Fronteiras". In. P. Poutignat, J. Streiffe-Fenart, Teorias da Etnicidade, São Paulo: Fundação Editora UNESP.

BECKER, Howard. Les photographies disent-elles la vérité ? Ethnologie française, 2007/1, tome XXXVII, p. 33-42.

BRITO, Celso. 2011. Capoeira Angola "glocalizada": análise etnográfica de um grupo da cidade de Lyon, França. In: Reunião de Antropologia do Mercosul 11. Curitiba. 
BOURDIEU, Pierre. L'illusion biographique. Actes de la recherche en sciences sociales, juin 1986, vol. 62/63, p. 69-72.

. Langage et Pouvoir Symbolique. $2^{\mathrm{e}}$ éd. Paris : Seuil, 2001 [1991].

. Questions de Sociologie. $2^{\mathrm{e}}$ éd. Paris : Éditions de Minuit, 2002 [1982].

BUTLER, Judith. 'Performative Acts and Gender Constitution: An Essay in Phenomenology and Feminist Theory. Theatre Journal, 1988, vol.4, p.519-31.

Gender trouble - feminisme and the subvertion of identity. London : Routledge, 1990.

CONORD, Sylvaine. Usages et fonctions de la photographie. Ethnologie française, 2007/1, vol.37, p.11-22.

DELAMONT, Sara et STEPHENS, Neil. 2008. Up on the roof: the embodied habitus of diasporic capoeira. Cultural Sociology 2(1): 57-74.

FERREIRA, Daniel Granada. 2005. La capoeira du Brésil à Paris.. MA Thesis. Paris: IHEAL Institut des Hautes Études de l'Amérique Latine, Université de Paris III Sorbonne Nouvelle.

2007. A capoeira do Brasil até Paris: redes sociais, transformações e adaptações da

prática da capoeira no Brasil e na França. In: CEISAL V CONGRESO EUROPEO CEISAL. Bruxelas. Disponível em: <http://www.reseau-amerique-latine.fr/ceisal-bruxelles/MS-MIG/MSMIG-2-Granada.pdf> Acesso em: 27 mar. 2012.

2008. Adaptação em Movimento: O processo de "transnacionalização" da capoeira na França. Revista Antropolítica (24): 63-86.

2010. "Fazer o bem, faz bem": Notas sobre identidade e o aprendizado da capoeira na França. Revista da Fundarte (20): 39-43

2015. Pratique de la capoeira em France et au Royaume Uni. Paris. Harmattan, 272p.

2015a. Tornar-se Mestre de capoeira em Londres. Mestre Fantasma e a relocalização da capoeira na Europa. Revista Antropolítica (UFF), v. 38, p. 298-324, 2015.

GLICK SCHILLER, Nina; LEVITT, Peggy. 2004. Conceptualizing Simultaneity: A Transnational Social Field Perspective on Society. International Migration Review 38 (3): 1002-1039.

GLICK-SCHILLER, Nina. 2010. A global perspective on transnational migration: Theorising migration without methodological nationalism. Diaspora and Transnationalism. In: T. Faist \& R. Bauböck (orgs.). Amsterdam: Amsterdam University Press: 109-130.

GRAVINA, Heloisa. 2010. Por cima do mar eu vim, por cima do mar eu vou voltar. Ph.D Dissertation. Porto Alegre, RS: Universidade Federal do Rio Grande do Sul.

GUIZARDI, Menara Lube. 2011. Todo lo que la boca come. Flujos, rupturas y fricciones de la capoeira en Madrid. Ph.D Dissertation. Madrid: Universidad Autonoma de Madrid.

. Para pensar las redes transnacionales: itinerarios e historias migratorias de los capoeiristas brasileños en Madrid. Vibrant, Brasília, v. 10, n. 2, Dec. 2013.

HANNERZ, Ulf. 1996. Transnational Connections. London: Routledge.

HINE, Christine. Virtual Ethnography. London: Sage, 2000.

JOSEPH, Janelle. 2008. Going to Brazil': transnational and corporeal movements of a Canadian-Brazilian martial arts community. Global Networks 8(2): 194-213.

2008. The Logical Paradox of the Cultural Comodity: Selling an "Authentic" Afro-

Brazilian Martial Art in Canada. Sociology of Sport Journal 25 (a): 498-515.

MARCUS, George. Ethnography in/of the World System: The Emergence of Multi-Sited Ethnography. Annual Review of Anthropology, 1995, vol. 24, p. 95-117.

. Au-delà de Malinowski et après Writing Culture : à propos du futur de l'anthropologie culturelle et du malaise de l'ethnographie. Ethnographiques.org [en ligne]. 2002, no ${ }^{\circ}$. Disponible sur http://www.ethnographiques.org/2002/Marcus.html

MARGOLIS, Maxine. 1994. Little Brazil: Imigrantes brasileiros em Nova York. Campinas: Papirus.

2003. Na virada do milênio a imigração brasileira para os Estados Unidos. Fronteiras Cruzadas. In: A. C. B. Martes \& S. Fleischer (orgs.). São Paulo: Paz e Terra: 51-72.

MARTES, Ana Cristina Braga. 1999. Brasileiros nos Estados Unidos - um estudo sobre imigrantes em Massachusetts. São Paulo: Paz e Terra.

2003. Raça e etnicidade - Opções e Constrangimentos. Fronteiras Cruzadas. In: A. C.

B. Martes \& S. Fleischer (orgs.). São Paulo: Paz e Terra: 73-98. 
PATARRA, Neide Lopes. 2005. Migrações internacionais de e para o Brasil contemporâneo. São Paulo em Perspectiva 19(3): 23-33.

PENHA, Marcelo Montes 2001. African Heritage and National Representation: Two cases of Brasilidade in New York City. Raizes e Rumos 7: 372-388.

REIS, Rossana Rocha e SALES, Teresa (orgs.). 1999. Cenas de um Brasil migrante. São Paulo: Boitempo.

RIBEIRO, Gustavo Lins. 1999. 'O que faz o Brasil, Brazil: Jogos identitários em São Francisco'. Cenas do Brasil Migrante. São Paulo: Boitempo: 45-85.

SALES, Teresa. 1999. Brasileiros longe de casa. São Paulo: Cortez. 2005. Eles vestem o avental da América. O Estado de São Paulo 20 mar. 2005(Caderno Aliás): J4-5.

TAYLOR, Gerard. Capoeira the jogo de angola from Luanda to the Cyberspace, Vol 2. Berkeley: Blue Snake books, 2007.

TRAVASSOS, Sonia Duarte. 2000. Capoeira, difusão e metamorfoses culturais entre Brasil e Estados Unidos. 2000. Ph.D Dissertation. Rio de Janeiro: Museu Nacional, Universidade Federal do Rio de Janeiro.

VASSALLO, Simone Ponde. 2001. Ethnicité, tradition et pouvoir : le jeu de la capoeira à Rio de Janeiro et à Paris. Ph.D Dissertation. Paris: EHESS École des Hautes Études en Sciences Sociales.

WESOLOWSKI, Katya. 2012. Professionalizing Capoeira: Politics of Play in Twenty-firstCentury Brazil. Latin American Perspectives 39(2): 82-89.

Recebido em: 30/01/2017. Aprovado em: 21/03/2017. 